\title{
Single-particle momentum distributions of Efimov states in mixed-species systems
}

\author{
M. T. Yamashita, ${ }^{1}$ F. F. Bellotti, ${ }^{2,3,4}$ T. Frederico, ${ }^{2}$ D. V. Fedorov, ${ }^{3}$ A. S. Jensen, ${ }^{3}$ and N. T. Zinner ${ }^{3}$ \\ ${ }^{1}$ Instituto de Física Teórica, UNESP-Univ Estadual Paulista, C.P. 70532-2, CEP 01156-970, São Paulo, SP, Brazil \\ ${ }^{2}$ Instituto Tecnológico de Aeronáutica, 12228-900, São José dos Campos, SP, Brazil \\ ${ }^{3}$ Department of Physics and Astronomy, Aarhus University, DK-8000 Aarhus C, Denmark \\ ${ }^{4}$ Instituto de Fomento e Coordenação Industrial, 12228-901, São José dos Campos, SP, Brazil
}

(Received 23 March 2013; published 4 June 2013)

\begin{abstract}
We solve the three-body bound-state problem in three dimensions for mass imbalanced systems of two identical bosons and a third particle in the universal limit where the interactions are assumed to be of zero range. The system displays the Efimov effect and we use the momentum-space wave equation to derive formulas for the scaling factor of the Efimov spectrum for any mass ratio assuming either that two or three of the two-body subsystems have a bound state at zero energy. We consider the single-particle momentum distribution analytically and numerically and analyze the tail of the momentum distribution to obtain the three-body contact parameter. Our findings demonstrate that the functional form of the three-body contact term depends on the mass ratio, and we obtain an analytic expression for this behavior. To exemplify our results, we consider mixtures of lithium with either two caesium or rubidium atoms which are systems of current experimental interest.
\end{abstract}

DOI: 10.1103/PhysRevA.87.062702

PACS number(s): 34.50.Cx, 03.65.Ge, 21.45.-v, 67.85.-d

\section{INTRODUCTION}

Few- and many-body systems in the presence of strong interactions is an increasingly fruitful direction in physics due to several advances in the experimental realization of such systems within cold atomic gases [1]. One aspect of this pursuit concerns the so-called unitary regime in which the two-body scattering amplitude saturates the unitarity bound of basic quantum mechanics. For neutral and nonpolar cold atomic gases, interactions are very short ranged and one can often use the universal limit where interactions are modeled by a zero-range potential which is then subsequently connected to the low-energy two-body scattering dynamics through the scattering length $a$. In this framework, the unitary regime is simply characterized by a scattering length that is much larger than any other relevant scale in the system under study. The problem of characterizing a strongly interacting system at unitarity in turn lacks a natural scale for the interactions, and one expects universal behavior of structural and dynamical properties that should be applicable irrespective of the details of the short-distance physics, and thus have applicability in many different subfields.

An important recent development was the derivation of a number of universal relations that describe the physics of unitary two-component Fermi gases by Tan [2]. It turns out that a parameter dubbed the (two-body) contact $C_{2}$ emerges in expressions for both structural (energy, adiabatic theorem, pressure, virial theorem [3]) and dynamical observables (inelastic loss [4], radio-frequency spectroscopy [5,6]). This provides a strong connection between the few-body quantities and many-body observables, a connection that has by now been experimentally verified [7,8]. The relations are not particular to the unitary Fermi gas but may also be applied to bosons [9-14] as another recent experimental effort has confirmed [15].

Another direction that has enjoyed access to the unitary regime is the study of universal three-body bound states and the famous Efimov effect [16]. The effect occurs close to the unitary point and implies that a sequence of three-body bound states occurs wherein two successive states always have the same fixed ratio of their binding energies. The effect was first observed in cold atomic gas experiments [17] and has subsequently opened a new research direction dubbed Efimov physics [18]. An interesting recent finding is that the presence of the Efimov effect implies an extension of the universal relations discussed above and the introduction of a three-body contact parameter $C_{3}[11,12]$. This parameter vanishes in the case of two-component Fermi gases since the Pauli principle suppresses three-body correlations at short distances [3].

In this paper, we study three-body bound Efimov-type states for systems that contain two identical bosons and a third distinguishable particle. Our goal is to address the contact parameters of such systems when the masses are different and for different strengths of the interaction parameters by computing the single-particle momentum distributions and studying their asymptotic behavior. We will consider only the universal regime, i.e., we approximate all two-body potentials by zero-range interactions. In comparison to the previous studies containing three identical bosons, we find that for different particles there are additional contributions to the asymptotic behavior of the momentum distributions. This suggests that such measurements are a useful probe to discriminate between effects of identical and nonidentical three-body correlations in the many current cold gas experiments that have mixtures of different kinds of atoms in the same trap.

The paper is organized as follows. In Sec. II, we introduce the momentum-space formalism that we use and Sec. III contains a discussion of the three-body wave function (or spectator function). In Sec. III, we also present an analytic derivation of the Efimov scaling factors as function of mass ratio in the cases where either two or three of the two-body subsystems have a two-body bound state at zero energy. We proceed to present our results for the asymptotic momentum distributions in Sec. IV and demonstrate that the subleading correction has a functional form that depends on the mass ratio. The details of the analytic derivations of the asymptotic momentum behavior are given in the Appendix for completeness. Results for relevant experimental mixtures 
of ${ }^{6} \mathrm{Li}^{1}{ }^{133} \mathrm{Cs}^{-133}{ }^{13} \mathrm{Cs}$ and ${ }^{6} \mathrm{Li}^{-}{ }^{87} \mathrm{Rb}-{ }^{87} \mathrm{Rb}$ are discussed in Sec. V for different choices of the interaction parameters. Section VI contains conclusions and outlook for future studies.

\section{FORMALISM}

We consider a system that has an $A A B$ structure, where the two $A$ particles are identical bosons and the third $B$ particle is of a different kind. When we discuss our results in the following, we will focus on two combinations that are of interest to current experimental efforts in cold atoms: $A={ }^{133} \mathrm{Cs}, B={ }^{6} \mathrm{Li}$ and $A={ }^{87} \mathrm{Rb}, B={ }^{6} \mathrm{Li}$.

Since we are interested in the universal limit where the range of the two-body potentials can be neglected, we consider purely zero-range interactions in the following. More precisely, if $r_{0}$ is the range of the two-body potential, we are assuming that the scattering length $a$ is $a \gg r_{0}$. For simplicity, we will use units where $\hbar=m_{A}=1$ from now on. After partial wave projection, the $s$-wave coupled subtracted integral equations for the spectator functions $\chi$ and the absolute value of the three-body binding energy $E_{3}$ are given by [19-21]

$$
\begin{aligned}
& \chi_{A A}(y)=2 \tau_{A A}\left(y ; E_{3}\right) \int_{0}^{\infty} d x \frac{x}{y} G_{1}\left(y, x ; E_{3}\right) \chi_{A B}(x), \\
& \chi_{A B}(y)=\tau_{A B}\left(y ; E_{3}\right) \int_{0}^{\infty} d x \frac{x}{y}\left[G_{1}\left(x, y ; E_{3}\right) \chi_{A A}(x)\right. \\
&\left.+\mathcal{A}_{2}\left(y, x ; E_{3}\right) \chi_{A B}(x)\right] ; \\
& \tau_{A A}\left(y ; E_{3}\right) \equiv \frac{1}{\pi}\left[\sqrt{E_{3}+\frac{\mathcal{A}+2}{4 \mathcal{A}} y^{2}} \mp \sqrt{E_{A A}}\right]^{-1}, \\
& \tau_{A B}\left(y ; E_{3}\right) \equiv \\
& \frac{1}{\pi}\left(\frac{\mathcal{A}+1}{2 \mathcal{A}}\right)^{3 / 2}\left[\sqrt{E_{3}+\frac{\mathcal{A}+2}{2(\mathcal{A}+1)} y^{2}}\right. \\
& G_{1}\left(y, x ; E_{3}\right) \equiv \log \frac{2 \mathcal{A}\left(E_{3}+x^{2}+x y\right)+y^{2}(\mathcal{A}+1)}{2 \mathcal{A}\left(E_{3}+x^{2}-x y\right)+y^{2}(\mathcal{A}+1)} \\
&-\log \frac{2 \mathcal{A}\left(\mu^{2}+x^{2}+x y\right)+y^{2}(\mathcal{A}+1)}{2 \mathcal{A}\left(\mu^{2}+x^{2}-x y\right)+y^{2}(\mathcal{A}+1)}, \\
&-\log \frac{2\left(\mathcal{A} E_{3}+x y\right)+\left(y^{2}+x^{2}\right)(\mathcal{A}+1)}{2\left(\mathcal{A} \mu^{2}-x y\right)+\left(y^{2}+x^{2}\right)(\mathcal{A}+1)} \\
& G_{2}\left(y, x ; E_{3}\right) \equiv \log \frac{\left(\mathcal{A} E_{3}-x y\right)+\left(y^{2}+x^{2}\right)(\mathcal{A}+1)}{2\left(y^{2}+x^{2}\right)(\mathcal{A}+1)}
\end{aligned}
$$

where $x$ and $y$ denote (dimensionless) momenta. We will use the natural logarithm throughout this paper, i.e. the one with base $e$. Here, we have introduced the mass number $\mathcal{A}=m_{B} / m_{A}$. The interaction energies of the $A A$ and $A B$ subsystems are parametrized by $E_{A A}$ and $E_{A B}$, and the plus and minus signs in (3) and (4) refer to virtual and bound two-body subsystems, respectively [22-24]. We map $E_{A A}$ and $E_{A B}$ into the usual scattering lengths $a_{A A}$ and $a_{A B}$ through the relation $E \propto|a|^{-2}$. This relation typically holds for broad resonances, and a more detailed mapping needs to be done in the general case [25]. Throughout most of this work we will focus on the region close to unitarity in the $A B$ system, i.e., $\left|a_{A B}\right| \rightarrow \infty$ or $E_{A B} \rightarrow 0$. In light of the fact that experimental information about mixed systems of the $A A B$ type is still sparse, we will consider the two extreme cases (i) $E_{A A}=0$ and (ii) a noninteracting $A A$ subsystem.

In the numerical work presented later on, we will set $\mu^{2}=1$ for the subtraction point (see for instance Ref. [21] for a detailed discussion and references). On the other hand, in the analytical derivations, we will take the limit $\mu \rightarrow \infty$. We note that this subtraction method is basically equivalent to the procedure employed by Danilov [26] to regularize the original three-body Skorniakov-Ter-Martirosian equation [27]. A very detailed recent discussion of these issues was given by Pricoupenko [28,29].

Defining as $\vec{k}_{\alpha}(\alpha=i, j, k)$ the momenta of each particle in the rest frame, we have that the Jacobi momenta from one particle to the center of mass of the other two and the relative momentum of the two are given, respectively, by

$$
\begin{aligned}
& \vec{q}_{k}=m_{i j, k}\left(\frac{\vec{k}_{k}}{m_{k}}-\frac{\vec{k}_{i}+\vec{k}_{j}}{m_{i}+m_{j}}\right)=\vec{k}_{k} \quad \text { and } \\
& \vec{p}_{k}=m_{i j}\left(\frac{\vec{k}_{i}}{m_{i}}-\frac{\vec{k}_{j}}{m_{j}}\right),
\end{aligned}
$$

where $\{i, j, k\}$ is an even permutation of the particles $\left\{A, A^{\prime}, B\right\}$ and we have used that $\vec{k}_{i}+\vec{k}_{j}+\vec{k}_{k}=0$ in the center-of-mass system. The reduced masses are defined such that $m_{i j}=\frac{m_{i} m_{j}}{m_{i}+m_{j}}$ and $m_{i j, k}=\frac{m_{k}\left(m_{i}+m_{j}\right)}{m_{i}+m_{j}+m_{k}}$.

In the following we define exactly what we mean by singleparticle momentum distributions for particles of types $A$ and $B$. For a zero-range potential, the three-body wave function for an $A A B$ system, composed by two identical particles $A$ and one different $B$, can be written in terms of the spectator functions in the basis $\left|\vec{q}_{B} \vec{p}_{B}\right\rangle$ as

$$
\begin{aligned}
\left\langle\vec{q}_{B} \vec{p}_{B} \mid \Psi\right\rangle & =\frac{\chi_{A A}\left(q_{i}\right)+\chi_{A B}\left(q_{j}\right)+\chi_{A B}\left(q_{k}\right)}{E_{3}+H_{0}} \\
& =\frac{\chi_{A A}\left(q_{B}\right)+\chi_{A B}\left(\left|\vec{p}_{B}-\frac{\vec{q}_{B}}{2}\right|\right)+\chi_{A B}\left(\left|\vec{p}_{B}+\frac{\vec{q}_{B}}{2}\right|\right)}{E_{3}+H_{0}},
\end{aligned}
$$

or in the basis $\left|\vec{q}_{A} \vec{p}_{A}\right\rangle$ as

$$
\begin{aligned}
& \left\langle\vec{q}_{A} \vec{p}_{A} \mid \Psi\right\rangle \\
& =\frac{\chi_{A A}\left(\left|\vec{p}_{A}-\frac{\mathcal{A}}{\mathcal{A}+1} \vec{q}_{A}\right|\right)+\chi_{A B}\left(\left|\vec{p}_{A}+\frac{1}{\mathcal{A}+1} \vec{q}_{A}\right|\right)+\chi_{A B}\left(q_{A}\right)}{E_{3}+H_{0}^{\prime}},
\end{aligned}
$$

where $H_{0}=\frac{p_{B}^{2}}{2 m_{A A}}+\frac{q_{B}^{2}}{2 m_{A A, B}}$ and $H_{0}^{\prime}=\frac{p_{A}^{2}}{2 m_{A B}}+\frac{q_{A}^{2}}{2 m_{A B, A}}$. The reduced masses are given by $m_{A A}=\frac{1}{2}, m_{A A, B}=\frac{2 \mathcal{A}}{\mathcal{A}+2}, m_{A B}=$ $\frac{\mathcal{A}}{\mathcal{A}+1}$, and $m_{A B, A}=\frac{\mathcal{A}+1}{\mathcal{A}+2}$.

The momentum distributions for the particles $A$ and $B$ are

$$
\begin{aligned}
& n\left(q_{B}\right)=\int d^{3} p_{B}\left|\left\langle\vec{q}_{B} \vec{p}_{B} \mid \Psi\right\rangle\right|^{2}, \\
& n\left(q_{A}\right)=\int d^{3} p_{A}\left|\left\langle\vec{q}_{A} \vec{p}_{A} \mid \Psi\right\rangle\right|^{2}
\end{aligned}
$$

and they are normalized such that $\int d^{3} q n(q)=1$. Note that our definition of momentum distributions as well as their normalizations differ from Ref. [12]. In Ref. [12] there is a 
factor of $1 /(2 \pi)^{3}$ multiplying the definition of $n(q)$, which is normalized to 3 , the number of particles.

\section{ASYMPTOTIC FORMULAS FOR THE SPECTATOR FUNCTIONS}

We now consider the asymptotic behavior of the spectator function to derive some analytic formulas and compare to corresponding numerical results. To access the large momentum regime $\sqrt{E_{3}} \ll q$, we take the limit $\mu \rightarrow \infty$ and $E_{3}=E_{A A}=$ $E_{A B} \rightarrow 0$. The coupled equations for the spectator functions consequently simplify and become

$$
\begin{aligned}
& \chi_{A A}(y)=\frac{2}{\pi}\left[y \sqrt{\frac{\mathcal{A}+2}{4 \mathcal{A}}}\right]^{-1} \int_{0}^{\infty} d x \frac{x}{y} G_{1 a}(y, x) \chi_{A B}(x) \\
& \chi_{A B}(y)= \frac{1}{\pi}\left(\frac{\mathcal{A}+1}{2 \mathcal{A}}\right)^{3 / 2}\left[y \sqrt{\frac{\mathcal{A}+2}{2(\mathcal{A}+1)}}\right]^{-1} \\
& \times \int_{0}^{\infty} d x \frac{x}{y}\left[G_{1 a}(x, y) \chi_{A A}(x)\right. \\
&\left.+\mathcal{A} G_{2 a}(y, x) \chi_{A B}(x)\right]
\end{aligned}
$$

where

$$
\begin{aligned}
G_{1 a}(y, x) & \equiv \log \frac{2 \mathcal{A}\left(x^{2}+x y\right)+y^{2}(\mathcal{A}+1)}{2 \mathcal{A}\left(x^{2}-x y\right)+y^{2}(\mathcal{A}+1)}, \\
G_{2 a}(y, x) & \equiv \log \frac{\left(y^{2}+x^{2}\right)(\mathcal{A}+1)+2 x y}{\left(y^{2}+x^{2}\right)(\mathcal{A}+1)-2 x y} .
\end{aligned}
$$

We now proceed to solve these equations by using the Ansätze

$$
\chi_{A A}(y)=c_{A A} y^{-2+i s} \quad \text { and } \quad \chi_{A B}(y)=c_{A B} y^{-2+i s},
$$

where $y$ once again denotes a (dimensionless) momentum. Inserting the functions (15) in the set of coupled equations and performing the scale transformation $x=y z$, in the integrand of Eqs. (11) and (12), one has the following set of equations:

$$
\begin{aligned}
& c_{A A}=c_{A B} \frac{2}{\pi} \sqrt{\frac{4 \mathcal{A}}{\mathcal{A}+2}} \\
& \times \int_{0}^{\infty} d z z^{-2+1+i s} \log \frac{2 \mathcal{A}\left(z^{2}+z\right)+(\mathcal{A}+1)}{2 \mathcal{A}\left(z^{2}-z\right)+(\mathcal{A}+1)}, \\
& c_{A B}=\frac{1}{\pi}\left(\frac{\mathcal{A}+1}{2 \mathcal{A}}\right)^{3 / 2} \sqrt{\frac{2(\mathcal{A}+1)}{\mathcal{A}+2}} \\
& \times \int_{0}^{\infty} d z z^{-2+1+i s}\left[c_{A A} \log \frac{2 \mathcal{A}(1+z)+z^{2}(\mathcal{A}+1)}{2 \mathcal{A}(1-z)+z^{2}(\mathcal{A}+1)}\right. \\
& \left.+\mathcal{A} c_{A B} \log \frac{\left(1+z^{2}\right)(\mathcal{A}+1)+2 z}{\left(1+z^{2}\right)(\mathcal{A}+1)-2 z}\right] \text {. }
\end{aligned}
$$

Inserting Eq. (16) into (17), the set of coupled equations can be written as a single transcendental equation

$$
\begin{aligned}
& \frac{1}{\pi}\left(\frac{\mathcal{A}+1}{2 \mathcal{A}}\right)^{3 / 2} \sqrt{\frac{2(\mathcal{A}+1)}{\mathcal{A}+2}} \\
& \quad \times\left(\mathcal{A} I_{1}(s)+\frac{2}{\pi} \sqrt{\frac{4 \mathcal{A}}{\mathcal{A}+2}} I_{2}(s) I_{3}(s)\right)=1,
\end{aligned}
$$

where we have defined

$$
\begin{aligned}
I_{1}(s) & =\int_{0}^{\infty} d z z^{-1+i s} \log \left[\frac{\left(z^{2}+1\right)(\mathcal{A}+1)+2 z}{\left(z^{2}+1\right)(\mathcal{A}+1)-2 z}\right] \\
& =\frac{2 \pi}{s} \frac{\sinh \left(\theta_{1} s-\frac{\pi}{2} s\right)}{\cosh \left(\frac{\pi}{2} s\right)}, \\
I_{2}(s) & =\int_{0}^{\infty} d z z^{-1+i s} \log \left[\frac{2 \mathcal{A}\left(z^{2}+z\right)+\mathcal{A}+1}{2 \mathcal{A}\left(z^{2}-z\right)+\mathcal{A}+1}\right] \\
& =\frac{2 \pi}{s} \frac{\sinh \left(\theta_{2} s-\frac{\pi}{2} s\right)}{\cosh \left(\frac{\pi}{2} s\right)}\left(\frac{\mathcal{A}+1}{2 \mathcal{A}}\right)^{i s / 2}, \\
I_{3}(s) & =\int_{0}^{\infty} d z z^{-1+i s} \log \left[\frac{2 \mathcal{A}(1+z)+(\mathcal{A}+1) z^{2}}{2 \mathcal{A}(1-z)+(\mathcal{A}+1) z^{2}}\right] \\
& =\frac{2 \pi}{s} \frac{\sinh \left(\theta_{2} s-\frac{\pi}{2} s\right)}{\cosh \left(\frac{\pi}{2} s\right)}\left(\frac{\mathcal{A}+1}{2 \mathcal{A}}\right)^{-i s / 2} \cdot
\end{aligned}
$$

The angles are given by the equations $\tan ^{2} \theta_{1}=\mathcal{A}(\mathcal{A}+2)$ and $\tan ^{2} \theta_{2}=(\mathcal{A}+2) / \mathcal{A}$ with the conditions that $\pi / 2<\theta_{1}, \theta_{2}<$ $\pi$. For the special case of equal masses, i.e., $\mathcal{A}=1$, we have $\theta_{1}=\theta_{2}, I_{1}=I_{2}=I_{3}$, and

$$
\left(\frac{1}{\pi} \sqrt{\frac{4}{3}} I_{1}(s)\right)+2\left(\frac{1}{\pi} \sqrt{\frac{4}{3}} I_{1}(s)\right)^{2}-1=0,
$$

for which the physically relevant solution is seen to be

$$
\frac{1}{\pi} \sqrt{\frac{4}{3}} I_{1}(s)=\frac{1}{2} .
$$

Using Eq. (19), we recover the celebrated Efimov equation for the scaling parameter $s$ of equal mass particles [16,30-32]. Another very interesting and relevant special case is when there is no interaction between the two $A$ particles, in which case we can set $c_{A A}=0$ in Eq. (17). The equation for the scale factor [Eq. (18)] now simplifies and we get

$$
\frac{\mathcal{A}}{\pi}\left(\frac{\mathcal{A}+1}{2 \mathcal{A}}\right)^{3 / 2} \sqrt{\frac{2(\mathcal{A}+1)}{\mathcal{A}+2}} I_{1}(s)=1 .
$$

This equation was first derived in Ref. [30] and later also discussed in Ref. [32]. The derivation of Eqs. (23) and (24) by using the asymptotic forms for the spectator functions reproduces the well-known results for the scaling parameter $s$. In Fig. 1, we plot the scaling factors $\exp (\pi / s)$ for the case when all three subsystems have resonant interaction, which is the expression in Eq. (18) valid for $E_{A A}=E_{A B}=0$ (solid line), and when there is no interaction in the $A A$ subsystem, which is the expression in Eq. (24) valid for $E_{A B}=0$ (dashed line). Our results are identical to those shown in Figs. 52 and 53 of Ref. [32].

What is important to notice is that for $m_{A} \gg m_{B}(\mathcal{A} \ll 1)$, the scaling factors are very similar, and both are much smaller than the equal mass case where $\mathcal{A}=1$. We can therefore see that in the $A A B$ system with heavy $A$ and light $B$, we should expect many universal three-body bound states ( $s$ large or equivalently $e^{\pi / s}$ small) irrespective of whether the heavyheavy subsystem is weakly or strongly interacting. Recent experiments with mixtures of ${ }^{6} \mathrm{Li}$ and ${ }^{133} \mathrm{Cs}$ indicate that there could be a resonance of the ${ }^{6} \mathrm{Li}^{-133} \mathrm{Cs}$ subsystem at a point where the scattering length in the ${ }^{133} \mathrm{Cs}-{ }^{133} \mathrm{Cs}$ system is close to zero, i.e., weak interaction in the $A A$ subsystem $[33,34]$. 


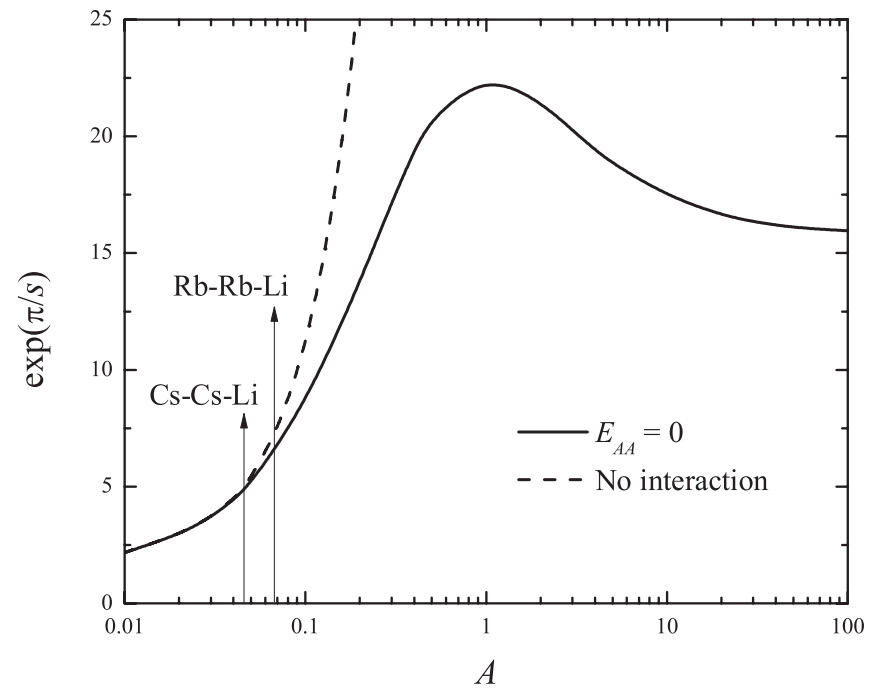

FIG. 1. Scaling parameter $s$ as a function of $\mathcal{A}=m_{B} / m_{A}$ for $E_{A A}=0$ and $E_{A B}=0$ (resonant interactions) (solid line) and for the situation where $E_{A B}=0$ but with no interaction between AA (dashed line). The arrows show the corresponding mass ratios for ${ }^{133} \mathrm{Cs}^{-}{ }^{133} \mathrm{Cs}-{ }^{6} \mathrm{Li}$ and ${ }^{87} \mathrm{Rb}-{ }^{87} \mathrm{Rb}-{ }^{6} \mathrm{Li}$.

Returning to Eqs. (11) and (12), there are two solutions which are complex conjugates of each other, i.e., $z^{ \pm i s}$. Apart from an overall normalization, there is still a relative phase between these two independent solutions. We determine this phase by requiring that the wave function be zero at a certain momentum denoted $q^{*}$. This parameter is known as the three-body parameter $[31,32]$. This is the momentumspace equivalent of the coordinate-space three-body parameter which is now believed to be simply related to the van der Waals two-body interaction of the atoms in question [35-43]. In this case, the asymptotic form of the spectator functions becomes

$$
\chi_{A A}(q)=c_{A A} q^{-2} \sin \left(s \log q / q^{*}\right) \text { and }
$$

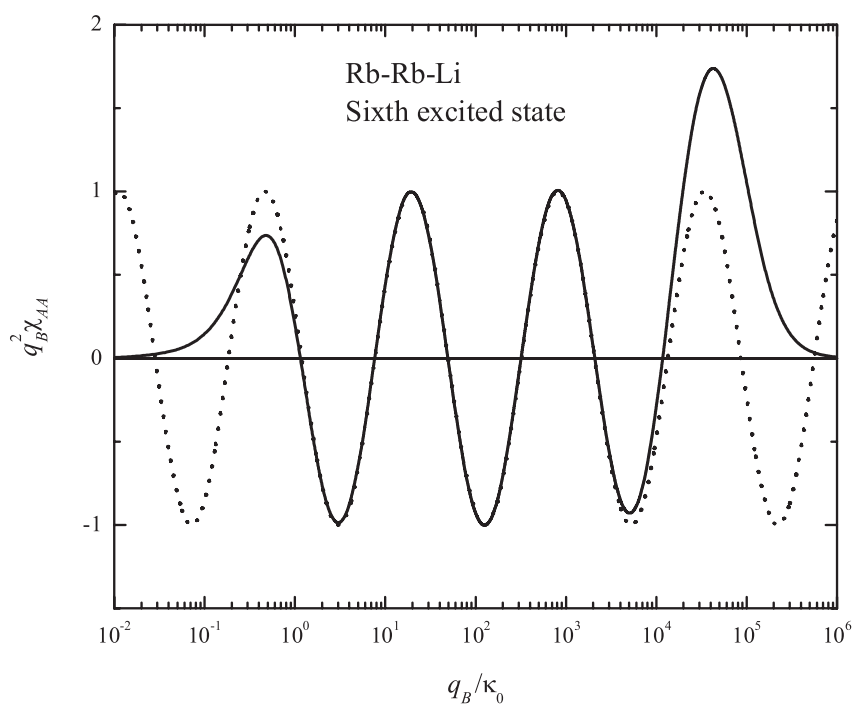

$$
\chi_{A B}(q)=c_{A B} q^{-2} \sin \left(s \log q / q^{*}\right) .
$$

Here, we use $q$ to denote momentum and we see that our boundary condition $\chi\left(q^{*}\right)=0$ is fulfilled. The asymptotic form of the spectator function should be compared with the solutions of the subtracted equations in the limit of large momentum, constrained by the window $\kappa_{0} \ll q_{B} \ll \mu$, where $\kappa_{0} \equiv \sqrt{E_{3}}$. The spectator functions $\chi_{A A}(q)$ for Rb-Rb-Li and Cs-Cs-Li compared to the respective asymptotic formula are shown in Fig. 2. In the idealized limit where $\kappa_{0}=0$ and $\mu \rightarrow \infty$, the two curves would coincide. We can thus see the effect of finite value of these two quantities on each end of the plots. The window of validity for the use of the asymptotic formulas, i.e., $\sqrt{E_{3}} \ll q \ll \mu$, can be clearly seen in these figures.

\section{ASYMPTOTIC MOMENTUM DENSITY}

In this section, we discuss the asymptotic momentum density for $n\left(q_{B}\right)$, i.e., the single-particle momentum distribution for the $B$ particle. From Eqs. (8) and (10), we can split the momentum density into nine terms, which can be reduced to four considering the symmetry between the two identical particles $A$. This simplifies the computation of the momentum density to the form

$$
n\left(q_{B}\right)=\sum_{i=1}^{4} n_{i}\left(q_{B}\right),
$$

where

$$
\begin{aligned}
n_{1}\left(q_{B}\right) & =\left|\chi_{A A}\left(q_{B}\right)\right|^{2} \int d^{3} p_{B} \frac{1}{\left(E_{3}+p_{B}^{2}+q_{B}^{2} \frac{\mathcal{A}+2}{4 \mathcal{A}}\right)^{2}} \\
& =\pi^{2} \frac{\left|\chi_{A A}\left(q_{B}\right)\right|^{2}}{\sqrt{E_{3}+q_{B}^{2} \frac{\mathcal{A}+2}{4 \mathcal{A}}}},
\end{aligned}
$$

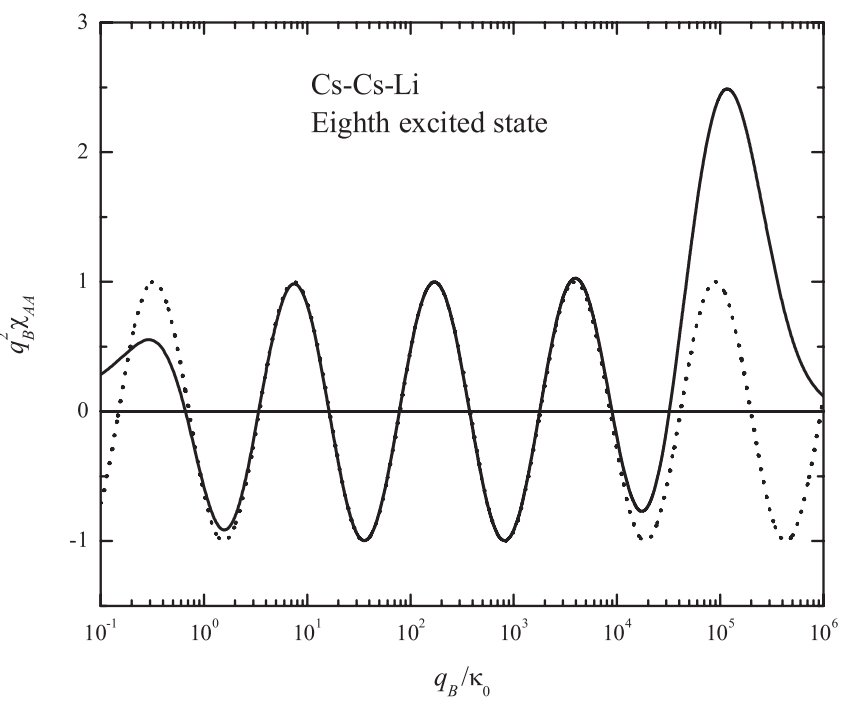

FIG. 2. Left: $\chi_{A A}(q)$ of the sixth excited state for $E_{A A}=E_{A B}=0, E_{3}=-8.6724 \times 10^{-12} E_{0}$, solution of the coupled equations (1) and (2) (solid line), compared with the asymptotic formula (15) for Rb-Rb-Li molecule (dotted line). Right: Same as left side for the eighth excited state of Cs-Cs-Li, $E_{3}=-8.9265 \times 10^{-13} E_{0}$. Here, we have defined $E_{0}=\hbar^{2} \mu^{2} / m_{A}$ and we work in units where $\hbar=m_{A}=\mu=1$ as explained in the text. 


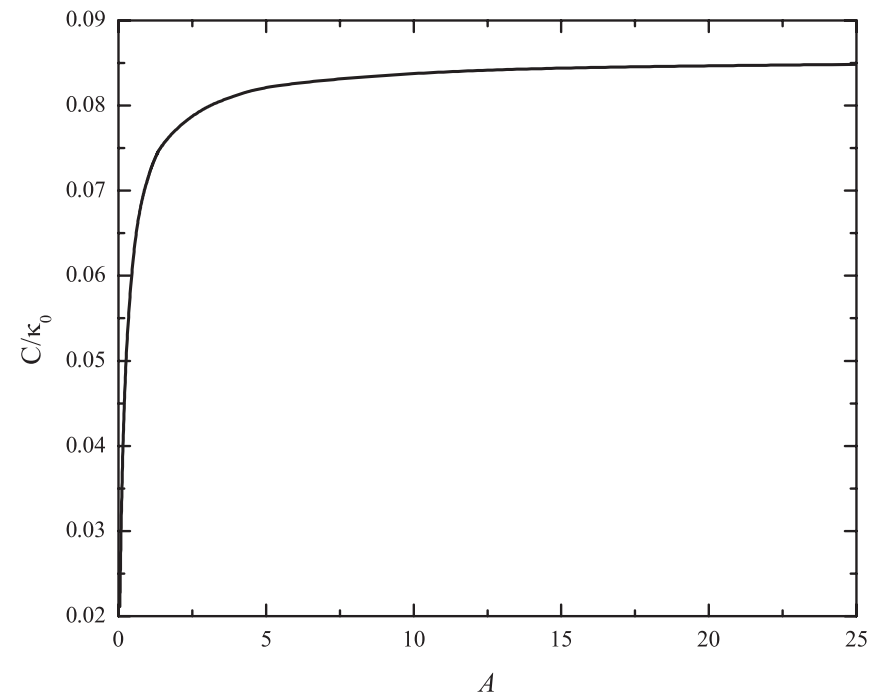

FIG. 3. $C / \kappa_{0}$ for mass ratios ranging from $\mathcal{A}=\frac{6}{133}$ to 25 . These results should be multiplied by the factor $3(2 \pi)^{3}$ in order to be compared with Ref. [12].

$$
\begin{aligned}
n_{2}\left(q_{B}\right) & =2 \int d^{3} p_{B} \frac{\left|\chi_{A B}\left(\left|\vec{p}_{B}-\frac{\vec{q}_{B}}{2}\right|\right)\right|^{2}}{\left(E_{3}+p_{B}^{2}+q_{B}^{2} \frac{\mathcal{A}+2}{4 \mathcal{A}}\right)^{2}} \\
& =2 \int d^{3} q_{A} \frac{\left|\chi_{A B}\left(q_{A}\right)\right|^{2}}{\left(E_{3}+q_{A}^{2}+\vec{q}_{A} \cdot \vec{q}_{B}+q_{B}^{2} \frac{\mathcal{A}+1}{2 \mathcal{A}}\right)^{2}}, \quad \text { (28) } \\
n_{3}\left(q_{B}\right) & =2 \chi_{A A}^{*}\left(q_{B}\right) \int d^{3} p_{B} \frac{\chi_{A B}\left(\left|\vec{p}_{B}-\frac{\vec{q}_{B}}{2}\right|\right)}{\left(E_{3}+p_{B}^{2}+q_{B}^{2} \frac{\mathcal{A}+2}{4 \mathcal{A}}\right)^{2}}+\text { c.c. }, \\
n_{4}\left(q_{B}\right) & =\int d^{3} p_{B} \frac{\chi_{A B}^{*}\left(\left|\vec{p}_{B}-\frac{\vec{q}_{B}}{2}\right|\right) \chi_{A B}\left(\left|\vec{p}_{B}+\frac{\vec{q}_{B}}{2}\right|\right)}{\left(E_{3}+p_{B}^{2}+q_{B}^{2} \frac{\mathcal{A}+2}{4 \mathcal{A}}\right)^{2}}+\text { c.c. }
\end{aligned}
$$

The leading-order term $\frac{C}{q_{B}^{4}}$ comes only from $n_{2}$ and the constant $C$ is simply given by $C=\frac{8 \mathcal{A}^{2}}{(\mathcal{A}+1)^{2}} \int d^{3} q_{A}\left|\chi_{A B}\left(q_{A}\right)\right|^{2}$. This formula gives $C / \kappa_{0}=0.0274$ for ${ }^{133} \mathrm{Cs}^{-133}{ }^{13} \mathrm{Cs}^{6} \mathrm{Li}$ and $C / \kappa_{0}=0.0211$ for ${ }^{87} \mathrm{Rb}^{8}{ }^{87} \mathrm{Rb}-{ }^{6} \mathrm{Li}$. For $\mathcal{A}=1$, we obtain $3(2 \pi)^{3} C / \kappa_{0}=53.197$, to be compared with the "exact" value 53.097 obtained in Ref. [12]. The factor $3(2 \pi)^{3}$ comes from the difference in choice of normalization. In Fig. 3, we plot the value of $C / \kappa_{0}$ for mass ratios ranging from $\mathcal{A}=\frac{6}{133}$ to 25 . The increase is very rapid until $\mathcal{A} \sim 5$ beyond which an almost constant value is reached. Note that we have used the second excited state to perform these calculations for $C$. This can explain the small discrepancy between the numerical and "exact" results, which was calculated for an arbitrary high excited state.

\section{A. Analysis of subleading terms}

In Ref. [12] it was shown that the nonoscillatory term of order $q_{B}^{-5}$ coming from $n_{1}$ to $n_{4}$ cancels for $\mathcal{A}=1$ (equal masses). Here, we will demonstrate that this conclusion does not hold for general $\mathcal{A} \neq 1$. Following, we will consider the $E_{3} \rightarrow 0$ limit, i.e., the three-body energy is assumed to be negligible, since we are interested in the imprint of Efimov states on the momentum distribution for excited Efimov states that are very extended and do not feel any short-range effects (as encoded in the three-body parameter $q^{*}$ discussed above).

(i) $n_{1}$ : Upon inserting (25) in the momentum distributions, we get for $n_{1}\left(q_{B}\right)$ the following asymptotic expression:

$$
\begin{aligned}
n_{1}\left(q_{B}\right) & \rightarrow 2 \pi^{2} \sqrt{\frac{\mathcal{A}}{\mathcal{A}+2}} \frac{\left|\chi_{A A}\left(q_{B}\right)\right|^{2}}{q_{B}} \\
& \rightarrow 2 \pi^{2}\left|c_{A A}\right|^{2} \sqrt{\frac{\mathcal{A}}{\mathcal{A}+2}} \frac{\left|\sin \left(s \log q_{B} / q^{*}\right)\right|^{2}}{q_{B}^{5}} .
\end{aligned}
$$

Averaging out the oscillating part yields $\frac{1}{2}$ and we have $\left\langle n_{1}\left(q_{B}\right)\right\rangle=\frac{\pi^{2}}{q_{B}^{5}}\left|c_{A A}\right|^{2} \sqrt{\frac{\mathcal{A}}{\mathcal{A}+2}}$.

(ii) $n_{2}$ : For large $q_{B}$, this term becomes

$$
\begin{aligned}
n_{2}\left(q_{B}\right)= & 2 \int d^{3} q_{A} \frac{\left|\chi_{A B}\left(q_{A}\right)\right|^{2}}{\left(q_{A}^{2}+\vec{q}_{A} \cdot \vec{q}_{B}+q_{B}^{2} \frac{\mathcal{A}+1}{2 \mathcal{A}}\right)^{2}} \\
= & \frac{8 \mathcal{A}^{2}}{q_{B}^{4}(\mathcal{A}+1)^{2}} \int d^{3} q_{A}\left|\chi_{A B}\left(q_{A}\right)\right|^{2} \\
& +\int d^{3} q_{A}\left|\chi_{A B}\left(q_{A}\right)\right|^{2}\left[\frac{2}{\left(q_{A}^{2}+\vec{q}_{A} \cdot \vec{q}_{B}+q_{B}^{2} \frac{\mathcal{A}+1}{2 \mathcal{A}}\right)^{2}}\right. \\
& \left.-\frac{8 \mathcal{A}^{2}}{(\mathcal{A}+1)^{2}} \frac{1}{q_{B}^{4}}\right],
\end{aligned}
$$

where we retain a subleading part since it is of the same order as the leading order of the other terms. This integral can be solved analytically (see the Appendix) and the final expression is

$$
\left\langle n_{2}\left(q_{B}\right)\right\rangle=-\frac{8 \pi^{2}\left|c_{A B}\right|^{2}}{q_{B}^{5}} \frac{\mathcal{A}^{3}(\mathcal{A}+3)}{(\mathcal{A}+1)^{3} \sqrt{\mathcal{A}(\mathcal{A}+2)}} .
$$

The special case $\mathcal{A}=1$ yields $\quad\left\langle n_{2}\left(q_{B}\right)\right\rangle=$ $-4 \pi^{2}\left|c_{A B}\right|^{2} /\left(\sqrt{3} q_{B}^{5}\right)$.

(iii) $n_{3}$ : The term for $n_{3}$ is considerably more complicated since it involves an angular integral. The details can be found in the Appendix and the final result is

$$
\begin{aligned}
\left\langle n_{3}\left(q_{B}\right)\right\rangle= & \frac{4 \pi^{2} c_{A A} c_{A B}}{q_{B}^{5} \cosh \left(\frac{s \pi}{2}\right)}\left\{\sqrt{\frac{\mathcal{A}}{\mathcal{A}+2}} \cos \left(s \log \sqrt{\frac{\mathcal{A}+1}{2 \mathcal{A}}}\right)\right. \\
& \times \cosh \left[s\left(\frac{\pi}{2}-\theta_{3}\right)\right]+\sin \left(s \log \sqrt{\frac{\mathcal{A}+1}{2 \mathcal{A}}}\right) \\
& \left.\times \sinh \left[s\left(\frac{\pi}{2}-\theta_{3}\right)\right]\right\},
\end{aligned}
$$

where $\quad \tan \theta_{3}=\sqrt{\frac{\mathcal{A}+2}{\mathcal{A}}}$ for $0 \leqslant \theta_{3} \leqslant \pi / 2$. The special case $\mathcal{A}=1$ yields $\theta_{3}=\pi / 3$ and $\left\langle n_{3}\left(q_{B}\right)\right\rangle=$ $4 \pi^{2}\left|c_{A A}\right|^{2} \cosh \left(\frac{s \pi}{6}\right) /\left[q_{B}^{5} \sqrt{3} \cosh \left(\frac{s \pi}{2}\right)\right]$.

(iv) $n_{4}$ : The $n_{4}$ term is also complicated by angular integrals and again we refer to the Appendix for details. The result is

$$
\begin{aligned}
\left\langle n_{4}\left(q_{B}\right)\right\rangle= & \frac{8 \pi^{2}\left|c_{A B}\right|^{2} \mathcal{A}^{2}}{s q_{B}^{5} \cosh \left(\frac{s \pi}{2}\right)}\left\{\sinh \left[s\left(\frac{\pi}{2}-\theta_{4}\right)\right]\right. \\
& \left.-\frac{s \mathcal{A}}{\sqrt{\mathcal{A}(\mathcal{A}+2)}(\mathcal{A}+1)} \cosh \left[s\left(\frac{\pi}{2}-\theta_{4}\right)\right]\right\},
\end{aligned}
$$



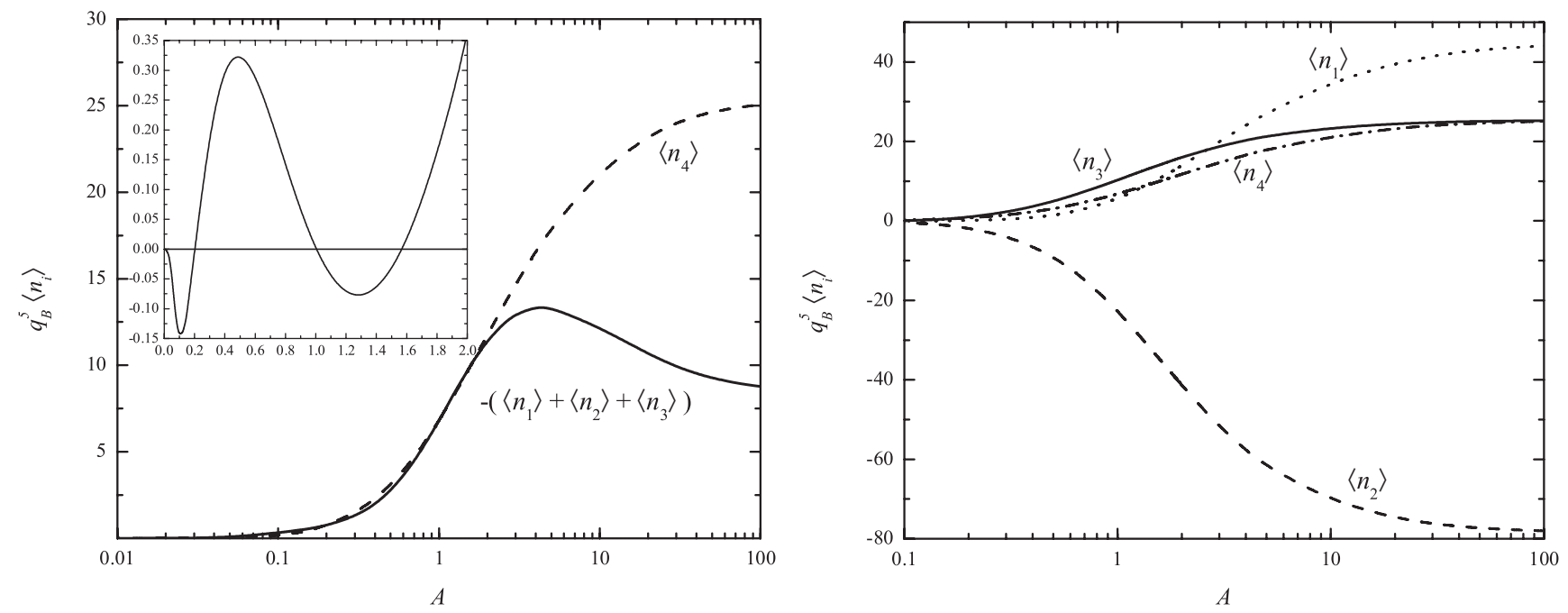

FIG. 4. (Left) Nonoscillatory contributions for $n_{1}+n_{2}+n_{3}$, and $n_{4}$ as function of the mass ratio $\mathcal{A}$. Their sum, shown in the inset on the left, cancels exactly for $\mathcal{A}=0.2,1$, and 1.57 . (Right) The individual contributions $n_{1}, n_{2}, n_{3}$, and $n_{4}$ as function of $\mathcal{A}$.

where $\tan \theta_{4}=\sqrt{\mathcal{A}(\mathcal{A}+2)}$ for $0 \leqslant \theta_{4} \leqslant \pi / 2$. The special case $\mathcal{A}=1$ yields $\theta_{4}=\pi / 3$ and $\left\langle n_{4}\left(q_{B}\right)\right\rangle=$ $8 \pi^{2}\left|c_{A A}\right|^{2}\left[\sinh \left(\frac{s \pi}{6}\right)-s /(2 \sqrt{3}) \cosh \left(\frac{s \pi}{6}\right)\right] /\left[s q_{B}^{5} \cosh \left(\frac{s \pi}{2}\right)\right]$.

It is important to note here that the asymptotic forms for the spectator functions have been used in the integrals where the integration is being performed from 0 to $\infty$. This may a priori cause problems for small momenta. However, a numerical check shows that the different behavior of the spectator functions at low momenta contributes only in an order higher than $q_{B}^{-5}$ to the integrals. This procedure is the same as that used in Ref. [12].

We now have analytic expressions for all the four terms in Eq. (26). The ratio between the coefficients $c_{A A}$ and $c_{A B}$ is given by Eq. (16), which can be used to eliminate one of these normalization factors. The other one can be determined from the overall normalization of the wave function, which we will not be concerned with here and we will merely set $c_{A B}=1$ from now on. In Fig. 4, we plot the contribution $-\left(n_{1}+n_{2}+n_{3}\right)$ and $n_{4}$ as a function of mass ratio $\mathcal{A}$ on the left-hand side and the individual contributions of $n_{1}, n_{2}, n_{3}$, and $n_{4}$ separately on the right-hand side. What is immediately seen is that for $\mathcal{A}=1$ we reproduce the result of Ref. [12], i.e., that the $q_{B}^{-5}$ nonoscillatory term cancels. However, for general $\mathcal{A}$ this is not the case and one should expect also a $q_{B}^{-5}$ term in the asymptotic momentum distribution for systems with two identical and a third particle when three-body bound states are present. This is the main result of our paper and it demonstrates that nonequal masses will generally influence not only the value of the contact parameter attributed to three-body bound states but also the functional form of the asymptotic momentum tail. Curiously, there is an oscillatory behavior around $\mathcal{A} \sim 1$ of the sum of all contributions. This is shown in the inset of Fig. 4 where we see zero crossings at $\mathcal{A}=0.2,1$, and 1.57. It seems quite clear that the oscillatory terms that all depend on the scale factor $s$ are to blame for this interesting behavior, but we have not found an easy analytic explanation for it. What makes this interesting is the fact that if we take ratios of typical isotopes of alkali atoms such as $\mathrm{Li}, \mathrm{Na}, \mathrm{K}, \mathrm{Rb}$, and $\mathrm{Cs}$, one can get rather close to 0.2 or 1.57 . For instance, taking one ${ }^{133} \mathrm{Cs}$ and two ${ }^{85} \mathrm{Rb}$ yields $\mathcal{A}=1.565$, while one ${ }^{7} \mathrm{Li}$ atom and two ${ }^{39} \mathrm{~K}$ atoms yields $\mathcal{A}=0.179$. These interesting ratios are thus close to experimentally accessible species.

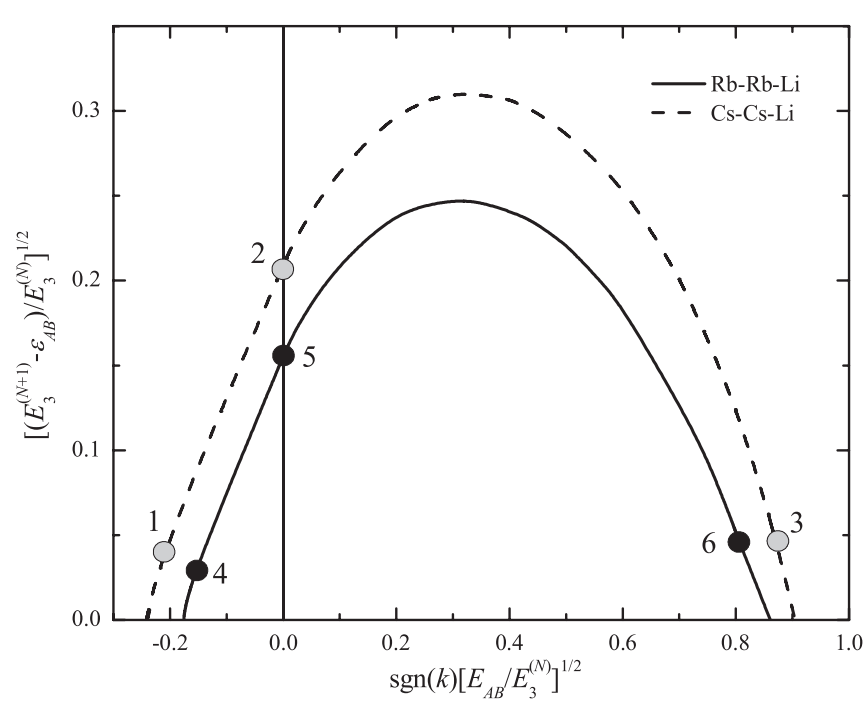

FIG. 5. Square root of the ratio of the $(N+1)$ th three-body state (measured from threshold $\epsilon_{A B}$ ) to the $N$ th state plotted as function of the square root of the ratio of $E_{A B}$ and the energy of the $N$ th threebody bound state. Note the factor $\operatorname{sgn}(k)$ which indicates whether the two-body system has a bound $(k=+1)$ or virtual $(k=-1)$ state. This sign is consistent with the convention introduced in Eqs. (3) and (4). The limit cycle, which should be in principle reached for $N \rightarrow \infty$, is achieved very fast so that the curve has been constructed using $N=2 . E_{A B}$ is the Cs-Li or Rb-Li two-body energy (Cs-Cs and $\mathrm{Rb}-\mathrm{Rb}$ two-body energies are zero). The negative and positive parts refer, respectively, to virtual and bound $A B$ states, such that $\epsilon_{A B}=0$ and $\epsilon_{A B} \equiv E_{A B}$, respectively, on the negative and positive sides. The circles labeled from 1 to 6 mark the points where the momentum distributions have been calculated. 

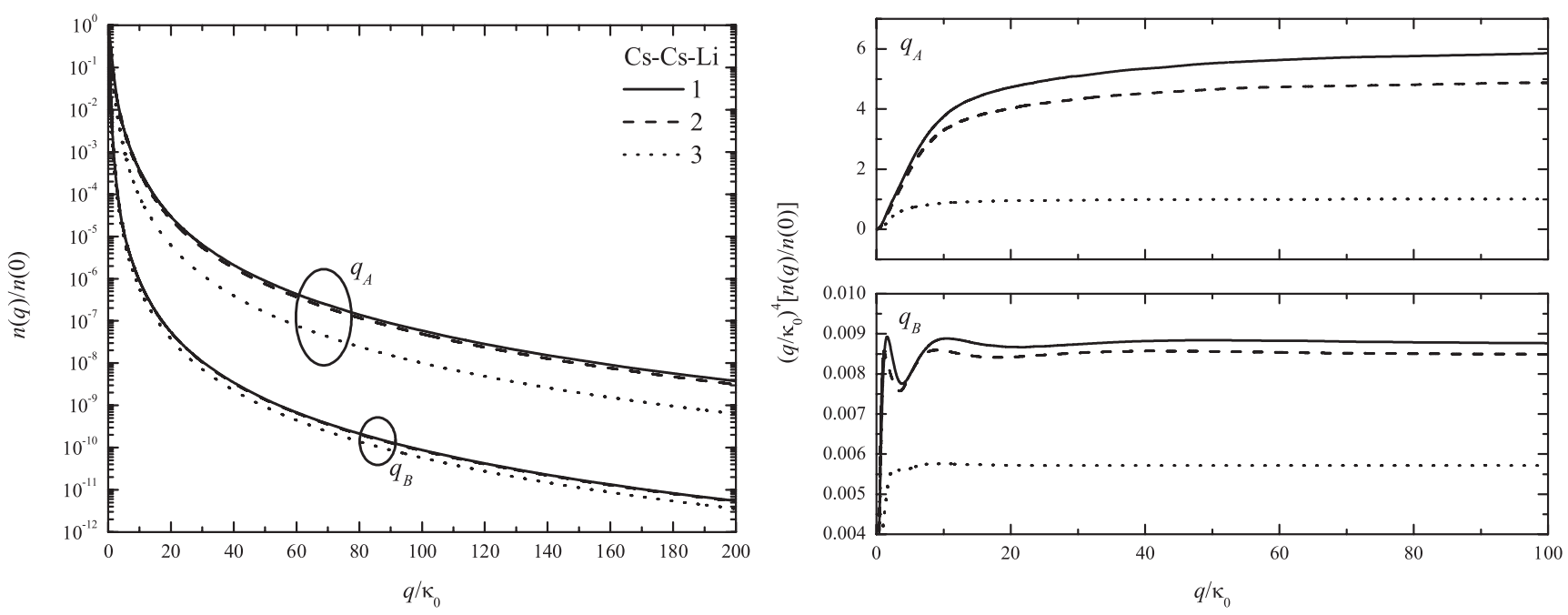

FIG. 6. Left: Momentum distribution for the second excited state as a function of the relative momentum of one ${ }^{133} \mathrm{Cs}\left(q_{A}\right)$ or ${ }^{6} \mathrm{Li}\left(q_{B}\right)$ to the center of mass of the remaining pair ${ }^{133} \mathrm{Cs}-{ }^{6} \mathrm{Li}$ or ${ }^{133} \mathrm{Cs}-{ }^{133} \mathrm{Cs}$. The solid, dashed, and dotted lines were calculated for the two- and three-body energies satisfying the ratios indicated by the points 1 to 3 in Fig. 5. The circles show the set of curves related to $q_{A}$ or $q_{B}$. Right: Same curves as on the left side multiplied by $q^{4}$, which shows explicitly the leading decay $1 / q^{4}$.

\section{NUMERICAL EXAMPLES}

We now provide some numerical examples of momentum distributions for the experimentally interesting systems with large mass ratios. We will focus on ${ }^{133} \mathrm{Cs}^{133}{ }^{13} \mathrm{Cs}-{ }^{6} \mathrm{Li}$ and ${ }^{87} \mathrm{Rb}-$ ${ }^{87} \mathrm{Rb}-{ }^{6} \mathrm{Li}$. Here, we will investigate two extreme possibilities: (i) the heavy-heavy subsystems, i.e., ${ }^{133} \mathrm{Cs}^{133} \mathrm{Cs}$ and ${ }^{87} \mathrm{Rb}-$ ${ }^{87} \mathrm{Rb}$, have a two-body bound state at zero energy and (ii) the opposite limit where they do not interact. In the first case, the heavy atoms are at a Feshbach resonance with infinite scattering length, while in the second case they are far from resonance and we assume a negligible background scattering length. As was recently demonstrated for the ${ }^{133} \mathrm{Cs}-$ ${ }^{6} \mathrm{Li}$ mixture, there are Feshbach resonances in the $\mathrm{Li}-\mathrm{Cs}$ subsystem at positions where the Cs-Cs scattering length is nonresonant $[33,34]$. While this does not automatically imply that the Cs-Cs channel can be neglected, we will make the assumption (ii) here. The formalism can be modified in a straightforward manner to also include interaction in the heavy-heavy subsystem.

As before, we denote the system $A A B$, where $A$ refers to the identical (bosonic) atoms ${ }^{133} \mathrm{Cs}$ or ${ }^{87} \mathrm{Rb}$, and $B$ to ${ }^{6} \mathrm{Li}$. By solving Eq. (18), one finds $s\left(\frac{6}{133}\right)=2.00588$ and $s\left(\frac{6}{87}\right)=$ 1.68334 when assuming that all three subsystems have large scattering lengths (solid line in Fig. 1). The situation where the interaction between the two identical particles is turned off is shown by the dashed line in Fig. 1. In this case, $s(\mathcal{A})$ was calculated from Eq. (17) by setting $c_{A A}=0$. This yields $s\left(\frac{6}{133}\right)=1.98572$ and $s(6 / 87)=1.63454$.
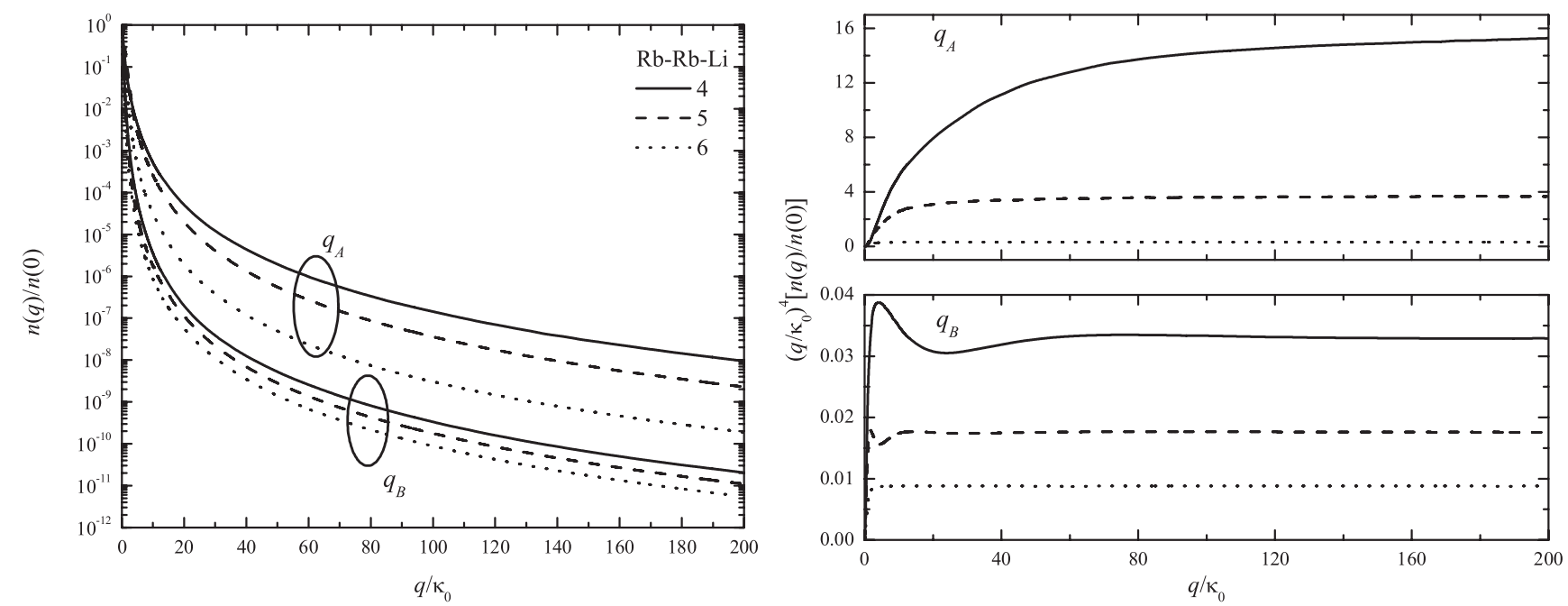

FIG. 7. Momentum distribution for the second excited state as a function of the relative momentum of one ${ }^{87} \mathrm{Rb}\left(q_{A}\right)$ or ${ }^{6} \mathrm{Li}\left(q_{B}\right)$ to the center of mass of the remaining pair ${ }^{87} \mathrm{Rb}-{ }^{6} \mathrm{Li}$ or ${ }^{87} \mathrm{Rb}^{87} \mathrm{Rb}$. The solid, dashed, and dotted lines were calculated for the two- and three-body energies satisfying the ratios indicated by the points 4 to 6 in Fig. 5. The circles show the set of curves related to $q_{A}$ or $q_{B}$. Right: same curves of the left side multiplied by $q^{4}$, which shows explicitly the leading decay $1 / q^{4}$. 


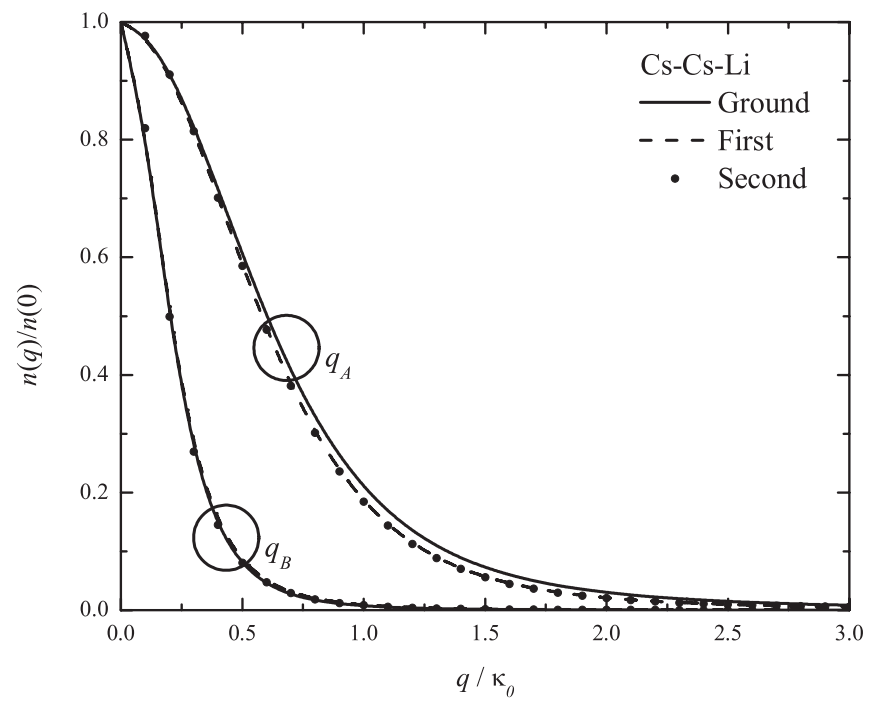

FIG. 8. Rescaled momentum distribution for the ground, first, and second excited states as a function of the relative momenta of ${ }^{133} \mathrm{Cs}$ to the center of mass of the pair ${ }^{6} \mathrm{Li}^{-133} \mathrm{Cs}\left(q_{A}\right)$ and ${ }^{6} \mathrm{Li}$ to the center of mass of the pair ${ }^{133} \mathrm{Cs}-{ }^{133} \mathrm{Cs}\left(q_{B}\right)$. The subsystem binding energies are all set to zero. Normalization to unity at zero momentum.

We first consider the binding energies. Assuming that the $\mathrm{Cs}-\mathrm{Cs}$ and $\mathrm{Rb}-\mathrm{Rb}$ two-body energies are zero, we have, for a system satisfying the universality condition $a \gg r_{0}$, that any observable should be a function of the remaining twoand three-body scales, which can be conveniently chosen as $E_{3}^{(N)}$ and $E_{A B}$ (the Cs-Li or Rb-Li two-body energy). Here, $N$ denotes the $N$ th consecutive three-body bound state with $N=0$ being the lowest one. Thus, the energy of an $N+1$ state can be plotted in terms of a scaling function relating only $E_{A B}$ and the previous state. The limit cycle, which should be in principle reached for $N \rightarrow \infty$, is achieved rapidly so

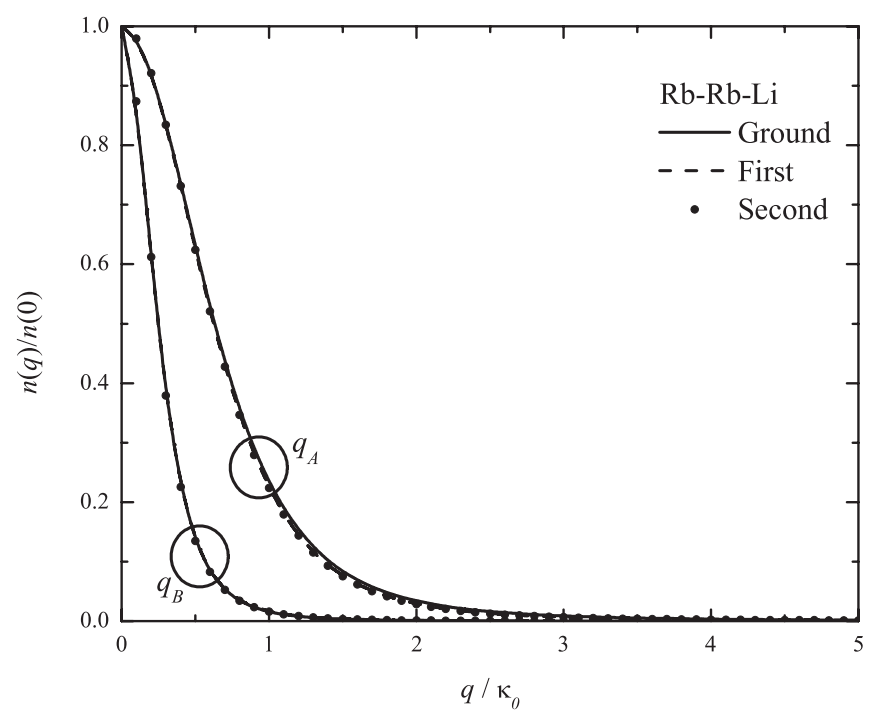

FIG. 9. Rescaled momentum distribution for the ground, first, second, and third excited states as a function of the relative momenta of ${ }^{87} \mathrm{Rb}$ to the center of mass of the pair ${ }^{87} \mathrm{Rb}-{ }^{6} \mathrm{Li}\left(q_{A}\right)$ and ${ }^{6} \mathrm{Li}$ to the center of mass of the pair ${ }^{87} \mathrm{Rb}^{87} \mathrm{Rb}\left(q_{B}\right)$. The subsystem binding energies are all set to zero. Normalization to unity at zero momentum. that we can construct the curve shown in Fig. 5 using $N=2$ $[21,22,44]$. The negative and positive parts of the horizontal axis refer, respectively, to virtual and bound two-body $A B$ states. The circles labeled from 1 to 6 mark the points where the momentum distributions have been calculated. The points 1 and 4 represent the Borromean case, the points 2 and 5 are the "Efimov situation," and in points 3 and $6 A B$ is bound.

Figures 6 and 7 give the momentum distributions of the second excited states for the energy ratio $\sqrt{E_{A B} / E_{3}}$ given by the points labeled from 1 to 6 in Fig. 5. According to our previous calculations [45], for fixed three-body energy, the size of the system increases as the number of bound two-body subsystems increase. Thus, it seems reasonable that the momentum distribution for the Borromean case (point 1) decreases slower. This behavior is clearly seen on the left side of Figs. 6 and 7. The distance of one atom to the center of mass of the other two is much larger for ${ }^{6} \mathrm{Li}$ than for ${ }^{133} \mathrm{Cs}$ or ${ }^{87} \mathrm{Rb}$, due to the large difference of the masses, such that the decrease of the momentum distribution for the heavier atom $\left(q_{A}\right.$ set) decreases much slower than that for the lighter one ( $q_{B}$ set). This also reflects on the momentum from which the leading-order decay $1 / q^{4}$ starts to be dominant. This difference becomes evident on the right side of Figs. 6 and 7, where we plotted $q^{4} n(q)$. Thus, the $q^{4}$ term is dominant above $(20-40) \kappa_{0}$ for $q_{b}$ and much slower for $q_{A}$ at about $(60-100) \kappa_{0}$.

Figures 8 and 9 show the rescaled momentum distributions for the ground, first, and second excited states. In these figures, the subsystem energies were chosen to zero, corresponding to the transition point to a Borromean configuration. In this situation, the only low-energy scale is $E_{3}$ (remember that the high-momentum scale is $\mu=1$ ). Therefore, in units in which $\mu=1$, to achieve a universal regime, in principle, to wash out the effect of the subtraction scale $\mu$, we have to go to a highly excited state (see, for instance, Fig. 2 and the comments inside the text associated to it). However, a universal lowenergy regime of $n\left(q_{B}\right) / n\left(q_{B}=0\right)$ is seen for momentum of the order of $\sqrt{E_{3}}$, even for the ground state which is smaller than excited states. Thus, in practice, the universal behavior of the momentum distribution is approached rapidly.

\section{CONCLUSIONS AND OUTLOOK}

In this work, we have calculated the single-particle momentum distribution of systems consisting of two identical bosonic particles and a third particle of a different kind with short-range interaction in the regime where three-body bound states and the Efimov effect occurs. We analytically calculate the asymptotic momentum distribution as a function of the mass ratio and find that the functional form is sensitive to this ratio. In the case of equal mass, we reproduce the results of Ref. [12], i.e., that the leading term has a $q^{-4}$ tail while the subleading contribution is $q^{-5}$ times a log-periodic oscillatory function that is characteristic of the Efimov effect and that depends on the scale factor (and thus on the mass ratio) of the Efimov states. In particular, we find that for general mass ratios, there is a nonoscillatory $q^{-5}$ contribution which appears to only vanish (and leave the oscillatory contribution behind) when the mass ratio is $0.2,1$, or 1.57 .

To exemplify our study, we consider ${ }^{133} \mathrm{Cs}-{ }^{133} \mathrm{Cs}-{ }^{6} \mathrm{Li}$ and ${ }^{87} \mathrm{Rb}^{8}{ }^{87} \mathrm{Rb}-{ }^{-} \mathrm{Li}$ where we numerically determine the coefficient 
of the $q^{-4}$ tail which is the two-body contact parameter introduced by Tan [2]. For these examples, we also numerically determine the momentum distributions of excited Efimov trimers for both the heavy and light components. Our numerical results demonstrate that the momentum distributions of ground, first, and second excited Efimov trimers approach universal behavior very fast at large but also at small momentum, indicating that one does not need to go to highly excited (and numerically challenging) three-body states in order to study the universal behavior of Efimov states in momentum space. Recent experiments have successfully measured the momentum distribution of ultracold atomic gases using time of flight and mapping to momentum space [7] and Bragg spectroscopy $[7,8,15]$. Observing a constant $1 / k^{5}$ contribution in a system with nonequal mass three-body states is a considerable challenge since one needs to first subtract the leading-order $1 / k^{4}$ contribution. The $1 / k^{4}$ tail can be extracted with good precision as discussed in the experimental papers $[7,8,15]$. If we assume that this subtraction of leading order can be done without severe increase of uncertainties in the data, then one would need to look at small and intermediate $k$ values for this subleading tail behavior.

A natural extension of this work is to consider three-body states in dimensions lower than three. In two dimensions, it is well known that no Efimov effect occurs [31,46-49] and, among other things, this implies that the momentum distribution does not have the subleading oscillatory behavior in two dimensions [50]. For two-dimensional systems with large differences in the masses, it is still possible to have many three-body bound states [51] and this should also be reflected in some way through the asymptotic momentum distribution. Another intriguing question is how the universal tail behavior and the contact relations behave in a crossover between twoand three-dimensional or one- and three-dimensional setups [29,50,52].
While we have studied only short-range interactions in this paper, it would be interesting to consider the momentum tails of few-body bound states in systems with long-range interactions. Recent experiments with heteronuclear molecules have demonstrated that the momentum distribution in dipolar systems can be probed using absorption imaging [53]. Fewbody bound states of dipolar particles have been predicted in a large parameter regime for both one- [54,55] and two-dimensional systems [56-59]. As was recently shown, one-dimensional dipolar few-body systems can in some cases be described by using zero-range interaction terms with appropriately chosen effective interactions parameters [60]. This opens up the possibility of using the same formalism with short-range interactions as discussed in this paper but applied in a one-dimensional setup. It should then be possible to derive the contact parameters in the presence of few-body bound states with dipolar particles in one dimension, similarly to what has been done for nondipolar bosons [61] and fermions [62].

\section{ACKNOWLEDGMENTS}

We thank R. Heck, J. Ulmanis, and R. Peres from the group of $\mathrm{M}$. Weidemüller in Heidelberg for updates on the experimental progress of ${ }^{6} \mathrm{Li}^{-}{ }^{133} \mathrm{Cs}$ mixed systems, and F. Werner for discussions on the work presented in Ref. [12]. M.T.Y. and T.F. thank the hospitality of the Department of Physics and Astronomy of the Aarhus University, where part of this work was done, and to the Brazilian agencies CNPq and FAPESP. This work was supported in part by a grant from the Danish Ministry of Science, Innovation, and Higher Education under the International Network program.

\section{APPENDIX: DERIVATION OF THE SUBLEADING TERMS}

\section{1. $n_{2}$ term}

In Eq. (32), we have a subleading term of the form

$$
\begin{aligned}
& \int d^{3} q_{A}\left|\chi_{A B}\left(q_{A}\right)\right|^{2}\left[\frac{2}{\left(q_{A}^{2}+\vec{q}_{A} \cdot \vec{q}_{B}+q_{B}^{2} \frac{\mathcal{A}+1}{2 \mathcal{A}}\right)^{2}}-\frac{8 \mathcal{A}^{2}}{(\mathcal{A}+1)^{2}} \frac{1}{q_{B}^{4}}\right] \\
& =\left|c_{A B}\right|^{2} \int \frac{d^{3} q_{A}}{q_{A}^{4}}\left[\frac{1}{\left(q_{A}^{2}+\vec{q}_{A} \cdot \vec{q}_{B}+q_{B}^{2} \frac{\mathcal{A}+1}{2 \mathcal{A}}\right)^{2}}-\frac{4 \mathcal{A}^{2}}{(\mathcal{A}+1)^{2}} \frac{1}{q_{B}^{4}}\right]=\frac{2 \pi\left|c_{A B}\right|^{2}}{q_{B}^{5}} \int \frac{d x}{x^{2}}\left[\frac{1}{x^{4}+\frac{1}{\mathcal{A}} x^{2}+\left(\frac{\mathcal{A}+1}{2 \mathcal{A}}\right)^{2}}-\frac{1}{\left(\frac{\mathcal{A}+1}{2 \mathcal{A}}\right)^{2}}\right],
\end{aligned}
$$

where in the first equality we have inserted the asymptotic form of $\left|\chi_{A B}\left(q_{A}\right)\right|^{2}=\left|c_{A B}\right|^{3} q_{A}^{-4} / 2$ obtained after averaging over the oscillatory term in Eq. (25). In the second equality, we have performed the angular integral and introduced the variable $q_{A}=q_{B} x$. We have also used the fact that the integrand is even to extend the integration to the entire real axis.

The function under the integral falls off faster than $1 / x$ for $|x| \rightarrow \infty$. We can therefore extend it to the complex domain and consider a contour in the upperhalf plane (or lower-half) that includes the real axis and a semicircle of large radius in a counterclockwise orientation. To use the residue theorem, we need to first find the poles of $f(x)$. Since $f(x)$ is regular at $x=0$, the only poles are out in the complex plane. The four poles are given by

$$
f(x)=\frac{1}{x^{2}}\left[\frac{1}{x^{4}+\frac{1}{\mathcal{A}} x^{2}+\left(\frac{\mathcal{A}+1}{2 \mathcal{A}}\right)^{2}}-\frac{1}{\left(\frac{\mathcal{A}+1}{2 \mathcal{A}}\right)^{2}}\right]
$$

$$
\begin{aligned}
& x_{1}=r e^{i \theta_{1} / 2}, \quad x_{2}=r e^{i\left(\pi-\theta_{1} / 2\right)}, \\
& x_{3}=r e^{i\left(\pi+\theta_{1} / 2\right)}, \quad x_{4}=r e^{-i \theta_{1} / 2},
\end{aligned}
$$


where $r=\sqrt{\frac{\mathcal{A}+1}{2 \mathcal{A}}}$ and $\tan ^{2} \theta_{1}=\mathcal{A}(\mathcal{A}+2)$. If we use the convention that $\pi / 2<\theta_{1}<\pi$ as in the main text, then $x_{1}$ and $x_{2}$ are the poles in the upper-half plane. The sum of the two residues is

$$
\operatorname{Res}\left(f, x_{1}\right)+\operatorname{Res}\left(f, x_{2}\right)=-\frac{1}{i r^{3}} \frac{\mathcal{A}(\mathcal{A}+3)}{(\mathcal{A}+1)^{2}} \frac{\cos \left(\frac{\theta_{1}}{2}\right)}{\sin \left(\theta_{1}\right)} .
$$

Using the residue theorem, the subleading term in Eq. (A1) then becomes

$$
\begin{aligned}
\int d^{3} q_{A}\left|\chi_{A B}\left(q_{A}\right)\right|^{2}\left[\frac{2}{\left(q_{A}^{2}+\vec{q}_{A} \cdot \vec{q}_{B}+q_{B}^{2} \frac{\mathcal{A}+1}{2 \mathcal{A}}\right)^{2}}\right. \\
\left.\quad-\frac{8 \mathcal{A}^{2}}{(\mathcal{A}+1)^{2}} \frac{1}{q_{B}^{4}}\right] \\
=-\frac{4 \pi^{2}\left|c_{A B}\right|^{2}}{q_{B}^{5} 2 \sin \left(\frac{\theta_{1}}{2}\right)} \frac{\mathcal{A}(\mathcal{A}+3)}{(\mathcal{A}+1)^{2}}\left(\frac{2 \mathcal{A}}{\mathcal{A}+1}\right)^{3 / 2} .
\end{aligned}
$$

From the definition of $\theta_{1}$ we see that $\cos \theta_{1}=-\frac{1}{\mathcal{A}+1}$ and $\left[2 \sin \left(\frac{\theta_{1}}{2}\right)\right]^{-1}=\sqrt{\frac{\mathcal{A}+1}{2(\mathcal{A}+2)}}$. The subleading term in $n_{2}$ is given by

$$
\left\langle n_{2}\left(q_{B}\right)\right\rangle=-\frac{8 \pi^{2}\left|c_{A B}\right|^{2}}{q_{B}^{5}} \frac{\mathcal{A}^{3}(\mathcal{A}+3)}{(\mathcal{A}+1)^{3} \sqrt{\mathcal{A}(\mathcal{A}+2)}},
$$

where the special case $\mathcal{A}=1$ yields $\theta_{1}=2 \pi / 3$ and $\left\langle n_{2}\right\rangle=$ $-4 \pi^{2}\left|c_{A B}\right|^{2} /\left(\sqrt{3} q_{B}^{5}\right)$.

\section{2. $n_{3}$ term}

Neglecting the three-body energy and making the variable transformation $\vec{q}_{A}=\vec{p}_{B}-\frac{\vec{q}_{B}}{2}$ in Eq. (29), we find

$n_{3}\left(q_{B}\right)=2 \chi_{A A}^{*}\left(q_{B}\right) \int d^{3} q_{A} \frac{\chi_{A B}\left(q_{A}\right)}{\left(q_{A}^{2}+\vec{q}_{A} \cdot \vec{q}_{B}+q_{B}^{2} \frac{\mathcal{A}+1}{2 \mathcal{A}}\right)^{2}}+$ c.c.

Defining $\vec{q}_{A}=q_{B} \vec{y}$, integrating over the solid angle, and replacing the asymptotic form for the spectator functions $\chi_{A A}$ and $\chi_{A B}$, given by Eq. (25), we get

$$
\begin{aligned}
n_{3}\left(q_{B}\right)= & 8 \pi \frac{c_{A A}^{*} c_{A B}}{q_{B}^{5}} \sin ^{2}\left(s \log q_{B} / q^{*}\right) \\
& \times \int_{0}^{\infty} \frac{\cos (s \log y) d y}{y^{4}+\frac{1}{\mathcal{A}} y^{2}+\left(\frac{\mathcal{A}+1}{2 \mathcal{A}}\right)^{2}} \\
& +8 \pi \frac{c_{A A}^{*} c_{A B}}{q_{B}^{5}} \sin \left(s \log q_{B} / q^{*}\right) \cos \left(s \log q_{B} / q^{*}\right) \\
& \times \int_{0}^{\infty} \frac{\sin (s \log y) d y}{y^{4}+\frac{1}{\mathcal{A}} y^{2}+\left(\frac{\mathcal{A}+1}{2 \mathcal{A}}\right)^{2}}+\text { c.c. }
\end{aligned}
$$

Averaging out the oscillatory terms, only the first term of Eq. (A8) gives a nonvanishing result

$$
\left\langle n_{3}\left(q_{B}\right)\right\rangle=4 \pi \frac{c_{A A}^{*} c_{A B}}{q_{B}^{5}} \int_{0}^{\infty} \frac{\cos (s \log y) d y}{y^{4}+\frac{1}{\mathcal{A}} y^{2}+\left(\frac{\mathcal{A}+1}{2 \mathcal{A}}\right)^{2}}+\text { c.c. }
$$

Expressing cosine in the complex exponential form we write that

$$
\begin{aligned}
I & =\int_{0}^{\infty} \frac{\cos (s \log y) d y}{y^{4}+\frac{1}{\mathcal{A}} y^{2}+\left(\frac{\mathcal{A}+1}{2 \mathcal{A}}\right)^{2}} \\
& =\operatorname{Re}\left[\int_{0}^{\infty} \frac{y^{i s} d y}{y^{4}+\frac{1}{\mathcal{A}} y^{2}+\left(\frac{\mathcal{A}+1}{2 \mathcal{A}}\right)^{2}}\right]=\operatorname{Re} I_{1},
\end{aligned}
$$

where $\mathrm{Re}$ denotes the real part. The residue theorem can be applied to solve the above integral. We set $y=e^{\alpha}$ in order to extend the interval of integration from $-\infty$ to $\infty$ and rewrite $I_{1}$ as

$$
I_{1}=\int_{-\infty}^{\infty} \frac{e^{\alpha(1+i s)}}{\left(e^{\alpha}-e^{\alpha_{1}}\right)\left(e^{\alpha}-e^{\alpha_{2}}\right)\left(e^{\alpha}-e^{\alpha_{3}}\right)\left(e^{\alpha}-e^{\alpha_{4}}\right)} d \alpha .
$$

The next steps are about extending the integrand $f(\alpha)$ to the complex plan, finding its poles, and evaluating the residues of the poles. All the roots in the denominator of $f(\alpha)$ are in the complex plane, out of the real axis, and are given by

$$
\begin{aligned}
& \alpha_{1}=\log r+i \theta_{3}, \quad \alpha_{2}=\log r-i\left(\pi-\theta_{3}\right), \\
& \alpha_{3}=\log r-i \theta_{3}, \quad \alpha_{4}=\log r+i\left(\pi-\theta_{3}\right),
\end{aligned}
$$

with $r=\sqrt{\frac{\mathcal{A}+1}{2 \mathcal{A}}}$ and $\tan \theta_{3}=\sqrt{\frac{\mathcal{A}+2}{\mathcal{A}}}$ for $0 \leqslant \theta_{3} \leqslant \pi / 2$.

We extend $f(\alpha)$ to the complex plane and choose the closed path as a rectangle of vertices $-R,+R,+R+i \pi$, and $-R+$ $i \pi$ (for $R \rightarrow \infty$ ), which encompasses the poles $\alpha_{1}$ and $\alpha_{4}$ in the upper-half plane. We are left with four integrals, namely, $J_{1}$ which extends along the real axis from $-R$ to $+R, J_{2}$ from $+R$ to $+R+i \pi, J_{3}$ from $+R+i \pi$ to $-R+i \pi$, and $J_{4}$ from $-R+i \pi$ to $-R$. In the limit $R \rightarrow \infty$, we find that $J_{1}=I_{1}$, $J_{3}=e^{-s \pi} I_{1}$, and $J_{3}$ and $J_{4} \rightarrow 0$. In this way, we find that

$$
\begin{aligned}
I_{1}= & \frac{2 \pi i}{1+e^{-\pi s}}\left[\operatorname{Res}\left(f, \alpha_{1}\right)+\operatorname{Res}\left(f, \alpha_{4}\right)\right] \\
= & \frac{\pi \mathcal{A}}{1+e^{-\pi s}} \sqrt{\frac{2}{(\mathcal{A}+2)(\mathcal{A}+1)}} \\
& \times\left(e^{i s\left(\log r+i \theta_{3}\right)-i \theta_{3}}+e^{i s\left[\log r+i\left(\pi-\theta_{3}\right)\right]+i \theta_{3}}\right),
\end{aligned}
$$

where $r$ and $\theta_{3}$ are defined after Eq. (A12).

It is necessary to split the real $\mathrm{Re}$ and imaginary Im parts to achieve our goal. Manipulating the trigonometric and hyperbolic functions we get

$$
\begin{aligned}
& \operatorname{Re} I_{1}=\frac{\pi}{2 \cosh \left(\frac{s \pi}{2}\right)}\left\{\sqrt{\frac{\mathcal{A}}{\mathcal{A}+2}} \cos (s \log r) \cosh \left[s\left(\frac{\pi}{2}-\theta_{3}\right)\right]+\sin (s \log r) \sinh \left[s\left(\frac{\pi}{2}-\theta_{3}\right)\right]\right\}, \\
& \operatorname{Im} I_{1}=\frac{-\pi}{2 \cosh \left(\frac{s \pi}{2}\right)}\left\{\sqrt{\frac{\mathcal{A}}{\mathcal{A}+2}} \sin (s \log r) \cosh \left[s\left(\frac{\pi}{2}-\theta_{3}\right)\right]+\cos (s \log r) \sinh \left[s\left(\frac{\pi}{2}-\theta_{3}\right)\right]\right\} .
\end{aligned}
$$


Finally, from Eqs. (A9), (A10), and (A14), the nonoscillating part of $n_{3}\left(q_{B}\right)$ is given by

$$
\left\langle n_{3}\left(q_{B}\right)\right\rangle=\frac{4 \pi^{2} c_{A A} c_{A B}}{q_{B}^{5} \cosh \left(\frac{s \pi}{2}\right)}\left\{\sqrt{\frac{\mathcal{A}}{\mathcal{A}+2}} \cos \left(s \log \sqrt{\frac{\mathcal{A}+1}{2 \mathcal{A}}}\right) \cosh \left[s\left(\frac{\pi}{2}-\theta_{3}\right)\right]+\sin \left(s \log \sqrt{\frac{\mathcal{A}+1}{2 \mathcal{A}}}\right) \sinh \left[s\left(\frac{\pi}{2}-\theta_{3}\right)\right]\right\},
$$

where $\tan \theta_{3}=\sqrt{\frac{\mathcal{A}+2}{\mathcal{A}}}$ for $0 \leqslant \theta_{3} \leqslant \pi / 2$. The special case $\mathcal{A}=1$ yields $\theta_{3}=\pi / 3$ and $\left\langle n_{3}\left(q_{B}\right)\right\rangle=4 \pi^{2}\left|c_{A A}\right|^{2} \cosh \left(\frac{s \pi}{6}\right) /$ $\left[q_{B}^{5} \sqrt{3} \cosh \left(\frac{s \pi}{2}\right)\right]$.

\section{3. $n_{4}$ term}

Although Eqs. (29) and (30) are similar, it is not possible to extend the results in the preceding section to obtain the nonoscillating term of $n_{4}\left(q_{B}\right)$. Defining $\vec{p}_{B}=\frac{\vec{q}_{B}}{2} \vec{y}$ and dropping the three-body energy, Eq. (30) becomes

$$
n_{4}\left(q_{B}\right)=\frac{4 \pi}{q_{B}} \int_{0}^{\infty} \frac{y^{2} d y}{\left(y^{2}+\frac{\mathcal{A}+2}{\mathcal{A}}\right)^{2}} \int_{-1}^{+1} d x \chi_{A B}^{*}\left(q_{B} x_{-}\right) \chi_{A B}\left(q_{B} x_{+}\right)+\text {c.c. }
$$

where $x_{ \pm}=\frac{1}{2} \sqrt{1+y^{2} \pm 2 y x}$. Replacing the spectator function by its asymptotic form (25) in the integral above, we are left with three terms, which read as

$$
\begin{aligned}
n_{4}\left(q_{B}\right)= & \frac{8 \pi\left|c_{A B}\right|^{2} \sin ^{2}\left(s \log \frac{q_{B}}{q^{*}}\right)}{q_{B}^{5}} \int_{0}^{\infty} \frac{y^{2} d y}{\left(y^{2}+\frac{\mathcal{A}+2}{\mathcal{A}}\right)^{2}} \int_{-1}^{+1} \frac{d x}{x_{+}^{2} x_{-}^{2}} \cos \left(s \log x_{+}\right) \cos \left(s \log x_{-}\right) \\
& +\frac{8 \pi\left|c_{A B}\right|^{2} \cos ^{2}\left(s \log \frac{q_{B}}{q^{*}}\right)}{q_{B}^{5}} \int_{0}^{\infty} \frac{y^{2} d y}{\left(y^{2}+\frac{\mathcal{A}+2}{\mathcal{A}}\right)^{2}} \int_{-1}^{+1} \frac{d x}{x_{+}^{2} x_{-}^{2}} \sin \left(s \log x_{+}\right) \sin \left(s \log x_{-}\right) \\
& +\frac{4 \pi\left|c_{A B}\right|^{2} \sin \left(s \log \frac{q_{B}}{q^{*}}\right) \cos \left(s \log \frac{q_{B}}{q^{*}}\right)}{q_{B}^{5}} \int_{0}^{\infty} \frac{y^{2} d y}{\left(y^{2}+\frac{\mathcal{A}+2}{\mathcal{A}}\right)^{2}} \int_{-1}^{+1} \frac{d x}{x_{+}^{2} x_{-}^{2}} \sin \left[s \log \left(x_{+} x_{-}\right)\right] .
\end{aligned}
$$

As it was done for $n_{3}\left(q_{B}\right)$, averaging out the oscillatory term, only the two first terms on the right-hand side of Eq. (A18) give a nonvanishing contribution. The angular integration is performed using that

$$
\int d x\left(\frac{\beta+x}{\beta-x}\right)^{ \pm i s / 2}\left(\beta^{2}-x^{2}\right)^{-1}= \pm\left(\frac{\beta+x}{\beta-x}\right)^{ \pm i s / 2}(i \beta s)^{-1}
$$

and the nonoscillating part of $n_{4}\left(q_{B}\right)$ is given by

$$
\left\langle n_{4}\left(q_{B}\right)\right\rangle=\frac{32 \pi\left|c_{A B}\right|^{2}}{i s q_{B}^{5}} \int_{0}^{\infty} \frac{y d y}{\left(y^{2}+\frac{\mathcal{A}+2}{\mathcal{A}}\right)^{2}\left(1+y^{2}\right)}\left[\left(\frac{y+1}{|y-1|}\right)^{i s}-\left(\frac{y+1}{|y-1|}\right)^{-i s}\right] .
$$

As was pointed out in [12], the absolute value complicates the calculation of this integral. Circumventing this problem, we follow the same trick as in [12], where the integral is split in two pieces: $y \in[0,1]$ and $y \in[1, \infty[$ and a new variable is introduced in each piece. We set $y=\frac{x-1}{x+1}$ in the first piece and $y=\frac{x+1}{x-1}$ in the second piece. Notice that in both cases $x \in[1, \infty[$. Now, we are able to apply the residue theorem to calculate the nonoscillating part of $n_{4}\left(q_{B}\right)$. First of all, we introduce a new variable $\alpha$, such that $x=e^{\alpha}$ and $\alpha \in[0, \infty$ [. As the resulting integrand is even, it allows us to extend the domain of integration to the entire real axis, i.e., $\alpha \in]-\infty, \infty[$. The integral in Eq. (A20) reads as

$$
\begin{aligned}
I & =\frac{i \mathcal{A}^{2}}{(\mathcal{A}+1)^{4}} \operatorname{Im}\left[\int_{-\infty}^{\infty} \frac{e^{\alpha(1+i s)}\left(e^{2 \alpha}-1\right)\left[(\mathcal{A}+1)^{2}\left(e^{6 \alpha}+1\right) / 2+\left(3 \mathcal{A}^{2}-2 \mathcal{A}-1\right)\left(e^{2 \alpha}+e^{4 \alpha}\right) / 2\right]}{\left(e^{\alpha}-e^{\alpha_{5}}\right)\left(e^{\alpha}-e^{\alpha_{6}}\right)\left[\left(e^{\alpha}-e^{\alpha_{1}}\right)\left(e^{\alpha}-e^{\alpha_{2}}\right)\left(e^{\alpha}-e^{\alpha_{3}}\right)\left(e^{\alpha}-e^{\alpha_{4}}\right)\right]^{2}} d \alpha\right] \\
& =\frac{i \mathcal{A}^{2}}{(\mathcal{A}+1)^{4}} \operatorname{Im} I_{1},
\end{aligned}
$$

where Im denotes the imaginary value. Extending the integrand $f(\alpha)$ to the complex plane, we find that all the roots in its denominator are on the imaginary axis and given by

$$
\alpha_{1}=i \theta_{4}, \quad \alpha_{2}=i\left(\pi-\theta_{4}\right), \quad \alpha_{3}=-i \theta_{4}, \quad \alpha_{4}=-i\left(\pi-\theta_{4}\right), \quad \alpha_{5}=i \frac{\pi}{2}, \quad \alpha_{6}=-i \frac{\pi}{2},
$$


where $\tan \theta_{4}=\sqrt{\mathcal{A}(\mathcal{A}+2)}$ for $0 \leqslant \theta_{4} \leqslant \frac{\pi}{2}$. Notice that $\alpha_{5}$ and $\alpha_{6}$ are simple poles while $\alpha_{1}, \alpha_{2}, \alpha_{3}$, and $\alpha_{4}$ are poles of second order [see Eq. (A22)].

To evaluate the contour integral, we choose the closed path as in the calculation of $n_{3}\left(q_{B}\right)$, namely, a rectangle of vertices $-R,+R,+R+i \pi$, and $-R+i \pi$ (for $R \rightarrow \infty$ ), which encompasses the poles $\alpha_{1}, \alpha_{2}$, and $\alpha_{5}$ in the upper-half plane. Once more, we are left with four integrals, i.e., $J_{1}$ which extends along the real axis from $-R$ to $+R, J_{2}$ from $+R$ to $+R+i \pi, J_{3}$ from $+R+i \pi$ to $-R+i \pi$, and $J_{4}$ from $-R+i \pi$ to $-R$. In the limit $R \rightarrow \infty$ we find that $J_{1}=I_{1}$, $J_{3}=e^{-s \pi} I_{1}$, and $J_{3}$ and $J_{4} \rightarrow 0$. In this way, we find that

$$
I_{1}=\frac{2 \pi i}{1+e^{-\pi s}}\left[\operatorname{Res}\left(f, \alpha_{1}\right)+\operatorname{Res}\left(f, \alpha_{2}\right)+\operatorname{Res}\left(f, \alpha_{5}\right)\right] \text {. }
$$

Calculating the residues is tedious, except for the case of $\alpha_{5}$ where $\operatorname{Res}\left(f, \alpha_{5}\right)=0$. After some algebraic work, the real and imaginary parts of $I_{1}$ are given by

$\operatorname{Re} I_{1}=\frac{\pi(\mathcal{A}+1)^{3} \mathcal{A}}{4 \sqrt{\mathcal{A}(\mathcal{A}+2)} \cosh \left(\frac{s \pi}{2}\right)} \cosh \left[s\left(\frac{\pi}{2}-\theta_{4}\right)\right]$,

$$
\begin{aligned}
\operatorname{Im} I_{1}= & \frac{\pi(\mathcal{A}+1)^{4}}{4 \sqrt{\mathcal{A}(\mathcal{A}+2)} \cosh \left(\frac{s \pi}{2}\right)} \\
& \times\left\{\sqrt{\mathcal{A}(\mathcal{A}+2)} \sinh \left[s\left(\frac{\pi}{2}-\theta_{4}\right)\right]\right. \\
& \left.-\frac{s \mathcal{A}}{\mathcal{A}+1} \cosh \left[s\left(\frac{\pi}{2}-\theta_{4}\right)\right]\right\} .
\end{aligned}
$$

Combining Eqs. (A20), (A22), and (A26), the nonoscillating part of $n_{4}\left(q_{B}\right)$ finally reads as

$$
\begin{aligned}
\left\langle n_{4}\left(q_{B}\right)\right\rangle= & \frac{8 \pi^{2}\left|c_{A B}\right|^{2} \mathcal{A}^{2}}{s q_{B}^{5} \cosh \left(\frac{s \pi}{2}\right)}\left\{\sinh \left[s\left(\frac{\pi}{2}-\theta_{4}\right)\right]\right. \\
& \left.-\frac{s \mathcal{A}}{\sqrt{\mathcal{A}(\mathcal{A}+2)}(\mathcal{A}+1)} \cosh \left[s\left(\frac{\pi}{2}-\theta_{4}\right)\right]\right\},
\end{aligned}
$$

where $\tan \theta_{4}=\sqrt{\mathcal{A}(\mathcal{A}+2)}$ for $0 \leqslant \theta_{4} \leqslant \pi / 2$. The special case $\mathcal{A}=1$ yields $\theta_{4}=\pi / 3$ and $\left\langle n_{4}\left(q_{B}\right)\right\rangle=$ $8 \pi^{2}\left|c_{A A}\right|^{2}\left[\sinh \left(\frac{s \pi}{6}\right)-s /(2 \sqrt{3}) \cosh \left(\frac{s \pi}{6}\right)\right] /\left[s q_{B}^{5} \cosh \left(\frac{s \pi}{2}\right)\right]$.
[1] I. Bloch, J. Dalibard, and W. Zwerger, Rev. Mod. Phys. 80, 885 (2008).

[2] S. Tan, Ann. Phys. (NY) 323, 2952 (2008); 323, 2971 (2008); 323, 2987 (2008).

[3] F. Werner and Y. Castin, Phys. Rev. A 86, 013626 (2012).

[4] E. Braaten and L. Platter, Phys. Rev. Lett. 100, 205301 (2008).

[5] E. Braaten, D. Kang, and L. Platter, Phys. Rev. Lett. 104, 223004 (2010).

[6] C. Langmack, M. Barth, W. Zwerger, and E. Braaten, Phys. Rev. Lett. 108, 060402 (2012).

[7] J. T. Stewart, J. P. Gaebler, T. E. Drake, and D. S. Jin, Phys. Rev. Lett. 104, 235301 (2010).

[8] E. D. Kuhnle, H. Hu, X. J. Liu, P. Dyke, M. Mark, P. D. Drummond, P. Hannaford, and C. J. Vale, Phys. Rev. Lett. 105, 070402 (2010).

[9] R. Combescot, F. Alzetto, and X. Leyronas, Phys. Rev. A 79, 053640 (2009).

[10] A. M. J. Schakel, arXiv:1007.3452v1.

[11] E. Braaten, D. Kang, and L. Platter, Phys. Rev. Lett. 106, 153005 (2011).

[12] Y. Castin and F. Werner, Phys. Rev. A 83, 063614 (2011).

[13] F. Werner and Y. Castin, Phys. Rev. A 86, 053633 (2012).

[14] M. Valiente, N. T. Zinner, and K. Mølmer, Phys. Rev. A 84, 063626 (2011).

[15] R. J. Wild, P. Makotyn, J. M. Pino, E. A. Cornell, and D. S. Jin, Phys. Rev. Lett. 108, 145305 (2012).

[16] V. Efimov, Yad. Fiz 12, 1080 (1970) [Sov. J. Nucl. Phys. 12, 589 (1971)].

[17] T. Kraemer et al., Nature (London) 440, 315 (2006).

[18] F. Ferlaino and R. Grimm, Physics 3, 9 (2010).

[19] A. E. A. Amorim, T. Frederico, and L. Tomio, Phys. Rev. C 56, R2378 (1997),

[20] F. Bellotti et al., J. Phys. B: At., Mol. Opt. Phys. 44, 205302 (2011).
[21] T. Frederico, A. Delfino, L. Tomio, and M. T. Yamashita, Prog. Part. Nucl. Phys. 67, 939 (2012).

[22] M. T. Yamashita, T. Frederico, A. Delfino, and L. Tomio, Phys. Rev. A 66, 052702 (2002).

[23] F. Bringas, M. T. Yamashita, and T. Frederico, Phys. Rev. A 69, 040702(R) (2004).

[24] M. T. Yamashita, T. Frederico, and L. Tomio, Few-Body Syst. 44, 191 (2008).

[25] C. Chin, R. Grimm, P. S. Julienne, and E. Tiesinga, Rev. Mod. Phys. 82, 1225 (2010).

[26] G. S. Danilov, Zh. Eksp. Teor. Fiz. 40, 698 (1961) [Sov. Phys.JETP 13, 349 (1961)].

[27] G. Skorniakov and K. Ter-Martirosian, Zh. Eksp. Teor. Fiz. 31, 775 (1956) [Sov. Phys.-JETP 4, 648 (1957)].

[28] L. Pricoupenko, Phys. Rev. A 82, 043633 (2010).

[29] L. Pricoupenko, Phys. Rev. A 83, 062711 (2011).

[30] A. S. Jensen and D. V. Fedorov, Europhys. Lett. 62, 336 (2003).

[31] E. Nielsen, D. V. Fedorov, A. S. Jensen, and E. Garrido, Phys. Rep. 347, 373 (2001).

[32] E. Braaten and H. W. Hammer, Phys. Rep. 428, 259 (2006).

[33] M. Repp, R. Pires, J. Ulmanis, R. Heck, E. D. Kuhnle, M. Weidemuller, and E. Tiemann, Phys. Rev. A 87, 010701 (2013).

[34] S. K. Tung, C. Parker, J. Johansen, C. Chin, Y. Wang, and P. S. Julienne, Phys. Rev. A 87, 010702(R) (2013).

[35] M. Berninger, A. Zenesini, B. Huang, W. Harm, H. C. Nagerl, F. Ferlaino, R. Grimm, P. S. Julienne, and J. M. Hutson, Phys. Rev. Lett. 107, 120401 (2011).

[36] P. Naidon, E. Hiyama, and M. Ueda, Phys. Rev. A 86, 012502 (2012).

[37] C. Chin, arXiv:1111.1484v2.

[38] R. Schmidt, S. P. Nath, and W. Zwerger, Eur. Phys. J. B 85, 386 (2012).

[39] J. Wang, J. P. D’Incao, B. D. Esry, and C. H. Greene, Phys. Rev. Lett. 108, 263001 (2012). 
[40] P. K. Sørensen, D. V. Fedorov, A. S. Jensen, and N. T. Zinner, Phys. Rev. A 86, 052516 (2012).

[41] P. Naidon, S. Endo, and M. Ueda, arXiv:1208.3912.

[42] Y. Wang, J. Wang, J. P. D'Incao, and C. H. Greene, Phys. Rev. Lett. 109, 243201 (2012).

[43] P. K. Sørensen, D. V. Fedorov, A. S. Jensen, and N. T. Zinner, J. Phys. B: At., Mol. Opt. Phys. 46, 075301 (2013).

[44] K. G. Wilson, Phys. Rev. D 3, 1818 (1971); S. D. Glazek and K. G. Wilson, Phys. Rev. Lett. 89, 230401 (2002); 92, 139901(E) (2004).

[45] M. T. Yamashita, L. Tomio, and T. Frederico, Nucl. Phys. A 735, 40 (2004).

[46] E. Nielsen, D. V. Fedorov, and A. S. Jensen, Phys. Rev. A 56, 3287 (1997).

[47] L. W. Bruch and J. A. Tjon, Phys. Rev. A 19, 425 (1979).

[48] S. K. Adhikari, A. Delfino, T. Frederico, I. D. Goldman, and L. Tomio, Phys. Rev. A 37, 3666 (1988); S. K. Adhikari, A. Delfino, T. Frederico, and L. Tomio, ibid. 47, 1093 (1993).

[49] A. G. Volosniev, D. V. Fedorov, A. S. Jensen, and N. T. Zinner, Eur. Phys. J. D 67, 95 (2013).

[50] F. F. Bellotti, T. Frederico, M. T. Yamashita, D. V. Fedorov, A. S. Jensen, and N. T. Zinner, Phys. Rev A 87, 013610 (2013).
[51] F. Bellotti et al., J. Phys. B: At., Mol. Opt. Phys. 46, 055301 (2013).

[52] M. Valiente, N. T. Zinner, and K. Mølmer, Phys. Rev. A 86, 043616 (2012).

[53] D. Wang, B. Neyenhuis, M. H. G. deMiranda, K. K. Ni, S. Ospelkaus, D. S. Jin, and J. Ye, Phys. Rev. A 81, 061404(R) (2010).

[54] M. Klawunn, J. Duhme, and L. Santos, Phys. Rev. A 81, 013604 (2010).

[55] N. T. Zinner, B. Wunsch, I. B. Mekhov, S. J. Huang, D. W. Wang, and E. Demler, Phys. Rev. A 84, 063606 (2011).

[56] D.-W. Wang, M. D. Lukin, and E. Demler, Phys. Rev. Lett. 97, 180413 (2006).

[57] M. Klawunn, A. Pikovski, and L. Santos, Phys. Rev. A 82, 044701 (2010).

[58] M. A. Baranov, A. Micheli, S. Ronen, and P. Zoller, Phys. Rev. A 83, 043602 (2011).

[59] N. T. Zinner, J. R. Armstrong, A. G. Volosniev, D. V. Fedorov, and A. S. Jensen, Few-Body Syst. 53, 369 (2012).

[60] A. G. Volosniev et al., New J. Phys. 15, 043046 (2013).

[61] M. Olshanii and V. Dunjko, Phys. Rev. Lett. 91, 090401 (2003).

[62] M. Barth and W. Zwerger, Ann. Phys. (NY) 326, 2544 (2011). 